livraisons

d'Histoire

de l'Architecture

\section{Livraisons de l'histoire de l'architecture}

$25 \mid 2013$

Architectures médiévales : types, matières et formes

\title{
Un architecte de la Ville de Paris Léon Salleron
}

(1820-1904)

Léon Salleron (1820-1904) architect of Paris

Léon Salleron (1820-1904) Architekt der Stadt Paris

\section{Geneviève Boisard}

\section{(2) OpenEdition}

Journals

Édition électronique

URL : http://journals.openedition.org/lha/314

DOI : $10.4000 /$ lha. 314

ISSN : 1960-5994

Éditeur

Association Livraisons d'histoire de l'architecture - LHA

Édition imprimée

Date de publication : 10 juin 2013

Pagination : 9-24

ISSN : 1627-4970

Référence électronique

Geneviève Boisard, «Un architecte de la Ville de Paris Léon Salleron (1820-1904) », Livraisons de I'histoire de l'architecture [En ligne], 25 | 2013, mis en ligne le 10 juin 2015, consulté le 30 avril 2019. URL : http://journals.openedition.org//ha/314 ; DOI : 10.4000//ha.314

Ce document a été généré automatiquement le 30 avril 2019.

Tous droits réservés à l'Association LHA 


\title{
Un architecte de la Ville de Paris Léon Salleron (1820-1904)
}

\author{
Léon Salleron (1820-1904) architect of Paris \\ Léon Salleron (1820-1904) Architekt der Stadt Paris
}

\section{Geneviève Boisard}

Parmi les nombreux architectes de la Ville qui, sous Haussmann et Baltard, donnèrent à Paris la physionomie qui est la sienne actuellement, Léon Salleron (1820-1904) n'est pas le plus connu. Architecte de nombreuses écoles et surtout de la mairie du XX arrondissement, son œuvre s'inscrit dans le style de l'époque et n'est pas d'une grande originalité. Les domaines modestes, mairie et écoles, dans lesquels s'exerça son activité à la Ville de Paris expliquent en partie cet effacement. Il n'eut pas de commandes prestigieuses, au contraire de Vaudremer, son contemporain. Modeste, il l'était également par son caractère et c'est seulement après sa retraite, en 1880 , qu'il posa sa candidature à la Société centrale des architectes, dont il fut membre du bureau pendant plusieurs années. Bien qu'il ait bénéficié d'une certaine notoriété de son vivant, il disparaît des publications, son dossier au centre de documentation du Musée d'Orsay est quasi inexistant. Il mérite cependant mieux que le relatif oubli dont il est victime et ses nombreuses réalisations justifient que l'on s'intéresse à ses travaux. Ont été privilégiées dans cet article les constructions privées moins connues, dont beaucoup subsistent encore, au détriment de ses réalisations officielles, dont de nombreuses reproductions ou photographies sont facilement accessibles.

\section{Les origines familiales}

2 Né le 29 décembre 1820, rue de Lourcine (actuellement rue Broca), dans la grande tannerie fondée par son grand père avant la Révolution, Claude-Augustin-Léon ${ }^{1}$ Salleron était fils d'Augustin Joseph et de Louise Mélanie Marcellot, d'une famille de riches marchands de bois. Il était issu d'une famille venue s'installer à Paris une cinquantaine d'années auparavant. Trois frères Salleron, originaires du baillage de Vitry-le-François, 
étaient venus à Paris dans les années 1770 pour exercer le métier de corroyeur. Deux d'entre eux, Joseph et Claude, y firent fortune, passant du corroyage à la tannerie, en fournissant les armées de la République, puis les armées impériales, profitant des confiscations révolutionnaires pour acquérir plusieurs installations sur les bords de la Bièvre. Leurs tanneries étaient parmi les plus importantes de Paris. Favorables aux idées de la Révolution, ils devinrent bonapartistes, libéraux sous la Restauration Devenus notables, le plus jeune, Claude, fut membre du Conseil général des manufacture sous l'Empire, député de la Seine sous la Restauration, Joseph, l'aîné fit prospérer ses affaires, l'un et l'autre furent décorés de la Légion d'honneur. Le fils de Joseph, Augustin Joseph, père de l'architecte Léon Salleron, fut moins heureux. Il avait le projet de réhabiliter les rives de la Bièvre, sur lesquelles se trouvaient les tanneries familiales, entraîna ses beauxfrères Marcellot dans une société d'investissement qui racheta de grands terrains et les nombreux moulins qui bordaient la rivière. Le succès escompté ne fut pas au rendez-vous. Malgré l'ouverture de plusieurs voies, la rue Pascal et la rue des Cordelières, sur leur grand domaine provenant de l'ancien couvent des Cordelière, les terrains et constructions ne se vendirent que lentement et à vil prix. Incapable d'honorer ses dettes, Augustin Joseph dut se déclarer en faillite en 1836. La grande tannerie du Moulin à tan des Cordelière fut vendue et Augustin Joseph devint gérant du Dépôt général des cuirs du commerce de la Boucherie de Paris qu'il avait fondé. Il dut démissionner de ses fonctions de maire du XII où il avait succédé à son frère. La maisonnée, composée des parents, des quatre enfants survivants d'une fratrie de onze et des domestiques, déménagea et s'installa dans une maison louée rue des Postes (actuellement rue Lhomond). Parmi les frères et sœur Élisabeth Hélène, l'ainée, âgée de 23 ans, donc majeure, échappe à la saisie ; les garçons sont plus jeunes. Léon a 15 ans, Félix, 13 et Paul, le dernier, 10.

\section{Une jeunesse austère}

3 À cette époque la faillite entraîne une véritable mort sociale. Léon Salleron, l'aîné des fils d'Augustin, était assez âgé pour ressentir douloureusement le changement de vie et de niveau social. Comme ses frères et ses cousins, il poursuivait ses études à la pension Savouré, dirigée par un allié de la famille, et au lycée Louis-le-Grand. Une fois bachelier, il s'inscrit à l'atelier de Duban pour préparer l'École des beaux-arts. Au premier abord un tel choix peut surprendre de la part d'un fils de négociant, mais il s'explique de bien des façons. Son père avait fait élever plusieurs constructions sur ses grands terrains, non seulement des maisons de rapport sur les rives de la Bièvre, mais deux réalisations plus importantes, tout d'abord la Maison d'éducation, réalisée en association avec Denis Cochin, ancien maire du XII ${ }^{e}$ arrondissement, en 1828, puis en 1830 il avait vendu à l'administration les terrains subsistant de l'ancien domaine des Cordelières pour créer une Maison de refuge et de travail pour l'extinction de la mendicité dans le département de la Seine devenue aujourd'hui l'Hôpital Broca. C'était l'époque où tout Paris était saisi d'une véritable fièvre de construction. Des quartiers entiers se créaient. Les grands terrains des anciennes communautés religieuses avec leurs grands espaces verts confisqués à la Révolution procuraient l'espace foncier disponible. Par ailleurs, en se destinant à la carrière d'architecte, Léon Salleron n'entre pas en territoire inconnu. Son père a travaillé avec régulièrement avec Jean-Joseph Rougevin, architecte, qui a fait pour lui les plans de la rue Pascal. Le frère aîné de ce dernier, Auguste Rougevin, sera le professeur de Léon à l'École des beaux-arts. 
Pour entrer à l'École, les aspirants architectes doivent tout d'abord s'inscrire dans un atelier pour s'y préparer et être formés sous la direction d'un maître. Léon s'inscrit dans l'atelier de Duban, qui a la réputation d'être romantique, bien qu'il ait évolué vers le classicisme. Le choix d'un atelier est déterminant car il oriente votre carrière. À cet égard il est intéressant de comparer les carrières de Léon Salleron et d'Émile Vaudremer, tous deux issus de familles de la bourgeoisie parisienne. Bien que séparés par une dizaine d'années, ils ont eu un parcours assez semblable et se sont côtoyés au service d'architecture de la Ville de Paris et dans de nombreuses commissions, mais Léon Salleron, formé par Duban, est resté romantique, attaché aux réminiscences de styles anciens quand il peut se laisser aller à ses goûts et n'est pas bridé par un programme trop strict, tandis que Vaudremer, formé par Abel Blouet, rationaliste, est un exemple de cette école.

Léon Salleron, jeune étudiant aux Beaux-arts

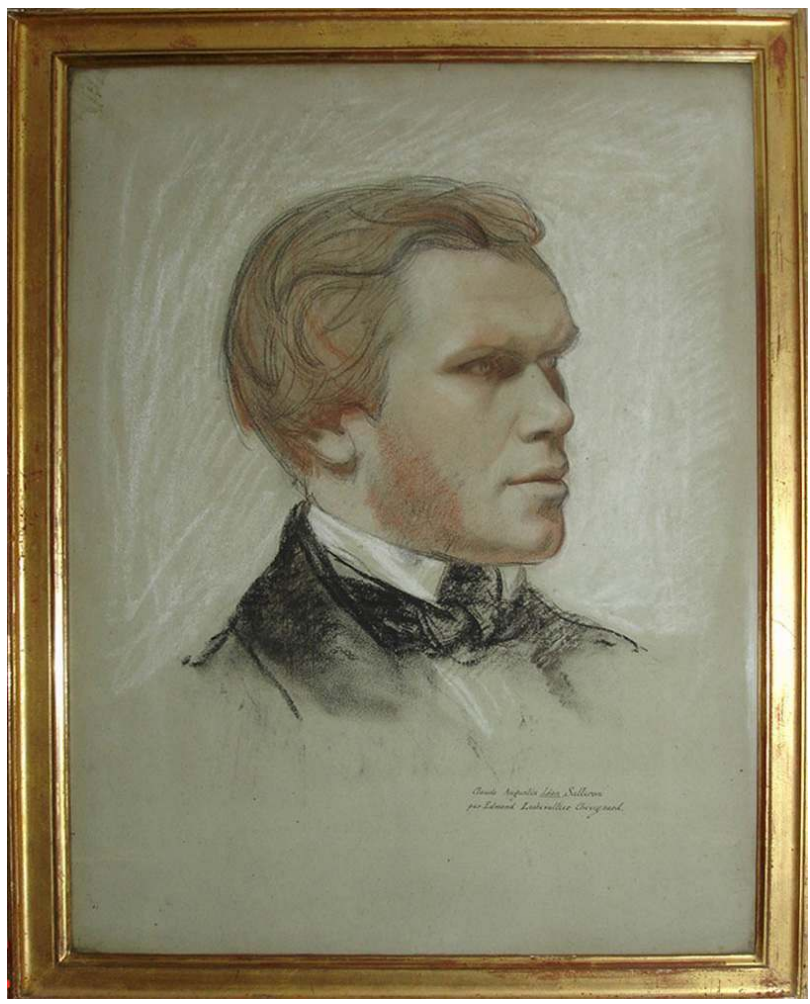

Par Lechevallier Chevignard. Collection particulière.

$\mathrm{Cl}$. de l'auteur

5 L'entrée à l'École des beaux-arts se fait sur concours avec trois épreuves : mathématiques, projection, architecture, qui permet d'être admis en $2^{\mathrm{e}}$ classe. Léon est admis sur liste temporaire le $1^{\text {er }}$ septembre 1842, puis sur concours le 23 décembre suivant. À cet égard il est amusant de noter qu'il fut signalé que trois des élèves de deuxième classe à l'École, Salleron, élève de Duban, Rimbault et Simonnet, élèves de Labrouste, « ont eu le mérite de s'écarter du programme du concours du 8 février 1843 » qui portait sur un château d'eau². $\mathrm{Au}$ cours de sa scolarité il recueille un certain nombre de distinctions en construction: première mention en maçonnerie en 1845 , deux premières mentions en construction générale et en fers en 1846, mentions en perspective, bois et architecture en 1847, deux mentions en architecture en 1848 et une première mention dans la même matière en 
1849. L'Académie d'architecture conserve toute une série de dessins aquarellés faits au cours de sa scolarité, de 1842 à 1849, qui manifestent l'influence de Duban sur son jeune élève. Ils ont été donnés à l'Académie en 1984 par Michel Homberg, architecte, petit-fils d'Eugène Théodore, gendre de Léon Salleron, dont il avait épousé la fille Thérèse en $1873^{3}$.

\section{Coupe sur un établissement de bains}

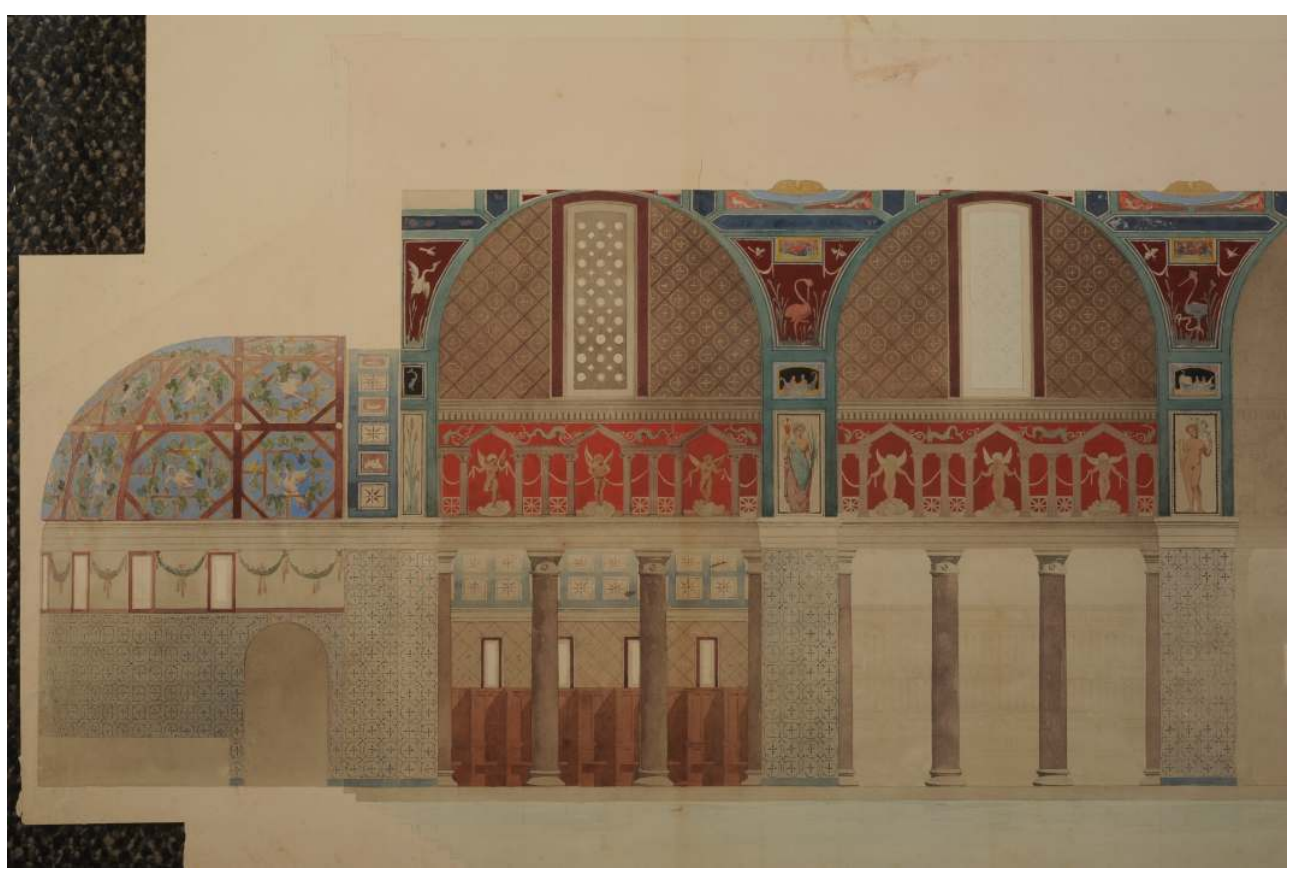

1844, signé L. Salleron, élève de Duban, Académie d'architecture

$\mathrm{Cl}$. Daniel Maunoury

Parallèlement à ses études aux Beaux-arts, Léon Salleron suit les cours de la jeune École centrale des arts et manufactures. En 1844 il obtient son diplôme d'ingénieur dans la catégorie constructeurs parmi les 33 nouveaux diplômés. « La sévérité des épreuves fait de ce diplôme une des plus honorables récompenses qu'un jeune homme puisse ambitionner à la fin de ses études $»^{4}$.

7 Il était entré dès 1842 au service d'architecture de la Ville de Paris en qualité de conducteur de travaux, ce qui lui permet de financer ses études car sa famille est alors dans la gêne. Il ne retrouvera une certaine fortune qu'après l'héritage de ses oncles Marcellot, vingt ans plus tard.

8 À la Ville de Paris il fera toute sa carrière, prenant sa retraite en 1879 en qualité d'architecte de $1^{\text {ère }}$ classe avec 35 ans et 6 mois de service. Bien que son dossier administratif n'ait pas été conservé aux Archives de Paris, on peut suivre sa carrière grâce l'Almanach royal, impérial puis national et par une note rédigée par ses soins le 21 mars 1885 à l'occasion de sa décoration de la Légion d'honneur.

\section{Une époque bâtisseuse qui voit la création des architectes municipaux}

9 Avant de retracer l'activité de Léon Salleron, il n'est pas inutile d'évoquer le contexte de l'époque. Comme il a déjà été dit, après la Révolution, une fois le calme revenu, le début 
du XIX' siècle est marqué par une fièvre constructrice. Dans les années suivantes, le second Empire veut moderniser Paris. Haussmann, préfet de la Seine de 1853 à 1870, va se lancer dans une transformation complète de la capitale à la fois pour des raisons d'hygiène et de sécurité publique. On se souvient en effet des révolutions de 1830 et de 1848 et il faut pouvoir lutter contre des émeutiers potentiels. L'année 1860 voit à la fois l'annexion des communes limitrophes et la réorganisation du Service d'architecture de la Ville de Paris. Haussmann crée alors un corps d'architectes municipaux qui ont un traitement fixe et ne sont plus payés au pourcentage. Auparavant les architectes travaillant pour la Ville n'étaient pas fonctionnaires. Choisis par le préfet, ils recevaient des honoraires et choisissaient leurs collaborateurs. La mairie avait seulement un service chargé d'entretenir les bâtiments, les constructions neuves étant confiées soit à des membres du service d'entretien, soit à des architectes de renon, qui devenaient alors membres du service temporaire. Les architectes étaient secondés par des inspecteurs chargés de l'inspection des chantiers du point de vue de la bonne exécution des travaux et de l'application du cahier des charges. À ceux-ci étaient eux-mêmes adjoints des conducteurs pour surveiller l'exécution des travaux. Les vérificateurs étaient chargés de rédiger les devis administratifs d'après les devis descriptifs de l'architecte et de tenir l'état sommaire mensuel des dépenses.

10 Lorsqu'il arrive à la préfecture, Haussmann crée donc un service d'architecture, qu'il confie à Baltard, protestant et ancien élève d'Henri-IV, comme lui. Il lui a d'ailleurs obtenu d'emporter la construction des Halles en lui faisant modifier son projet conformément aux souhaits de l'empereur. Baltard a alors sous ses ordres quatre architectes en chef et vingt architectes ordinaires. Contrairement aux règles, les architectes municipaux se voient le plus souvent confier la construction des édifices municipaux et passent alors au service temporaire. Après 1860 Haussmann divise le service ordinaire d'architecture (service permanent) en dix sections territoriales comprenant chacune deux arrondissements. Il les confie à dix architectes ordinaires, cinq choisis parmi les anciens et cinq nouveaux, grands prix de Rome. Ces sections passent à 14 en 1864, deux d'entre elles comprenant deux arrondissements, puis à vingt en 1866 . C'est alors que Léon Salleron, entré en 1843 comme conducteur, devenu sous-inspecteur, inspecteur puis architecte ordinaire, est chargé de la section du XX arrondissement. Il a alors 46 ans, avec vingt-trois ans d'ancienneté. En tant que responsable d'arrondissement il va être chargé de la construction de la mairie et de celle des nouvelles casernes d'octroi. Après la chute de l'Empire, la réforme de 1871 limite les attributions des architectes aux " travaux neufs de minime importance ${ }^{5}$. Salleron assure alors la construction des écoles de la rue Riblette (1873-1875), l'école maternelle de la rue Vitruve (1873), l'appropriation de l'école rue des Pyrénées (1875). Il n'est donc pas surprenant que, lors de la nouvelle réforme de 1877, qui répartit les attributions par type de bâtiments, il soit chargé avec Jean-Ferdinand Deconchy, comme architecte-adjoint, de la $\mathrm{VII}^{\mathrm{e}}$ section, écoles communales, asiles et orphelinats, car, comme ancien inspecteur de la $\mathrm{V}^{\mathrm{e}}$ section, chargée des établissements d'instruction communale, écoles et ouvroirs, il avait déjà acquis une certaine expérience en la matière. 


\section{Développement de l'enseignement et floraison des constructions d'écoles au XIX ${ }^{e}$ siècle}

11 Tout le XIX siècle a été une période de grande activité en matière de constructions scolaires. Pendant longtemps, l'enseignement avait été le monopole de l'école privée. Le décret du 17 mars 1808 prescrivait que « les Frères des écoles chrétiennes sont brevetés et encouragés par le grand maître de l'Université [...] Les supérieurs de cette congrégation sont membres de l'Université ». Cependant bientôt se manifeste une réaction contre cette mainmise cléricale. En 1830 le conseil municipal vote la création dans chaque quartier d'une école gratuite, laïque, mais aussi d'une école congréganiste pour les garçons et pour les filles. À cette époque, alors que les Frères des écoles chrétiennes pratiquent l'enseignement simultané où chaque frère se voit confier une cinquantaine d'élèves, la majeure partie des écoles pratique l'enseignement mutuel, où un seul instituteur dirige un très grand nombre d'élèves, aidé de moniteurs, qui sont les meilleurs élèves chargés d'aider leurs condisciples. En 1833 la loi Guizot précise que chaque commune doit entretenir au moins une école de garçons et choisit ses instituteurs parmi laïcs ou religieux. La loi Falloux de 1850 étend cette obligation aux filles dans les communes d'au moins 800 habitants. La défaite de 1870, interprétée comme la manifestation de la supériorité des instituteurs allemands sur les Français, provoque un sursaut. La loi Jules Ferry en 1878 proclame "l'instruction générale, obligatoire, gratuite et laïque ». Parallèlement toute une série de textes mettent en place l'organisation scolaire. Dès 1867 Octave Gréard, alors inspecteur à l'académie de Paris, impose le même programme à toutes les écoles primaires. Il devient en 1870 directeur de l'enseignement primaire du département de la Seine. Le 30 mars 1879 est créée une commission des bâtiments et du matériel scolaire. Léon Salleron figure parmi les cinq architectes qui la composent, aux côtés de Viollet-le-Duc, Narjoux, Trélat et Vaudremer. Cette commission devait élaborer un règlement pour la construction et l'ameublement de la maison d'école. Dissoute après la publication de son rapport en juin 1880, elle est remplacée par un comité des bâtiments scolaires, qui doit examiner les projets de province qui seront subventionnés. Le comité comprend neuf membres, dont Félix Narjoux, Eugène Train, Joseph-Antoine Bouvard et Léon Salleron, tous les quatre architectes de la Ville de Paris. À ce titre Léon Salleron fera partie de nombreux jurys de concours pour des établissements scolaires à Paris et en province. Il prend cette charge avec sérieux et avec une haute opinion de sa profession, car nous savons qu'en 1888 il refuse de faire partie du jury du concours d'Asnières parce que, sur les treize membres du jury, cinq seulement étaient architectes et parce que le programme laissait à l'administration municipale la faculté de laisser le soin de confier l'exécution à qui bon lui semblerait « ce qui réduit le rôle des architectes à une simple consultation ». Cette attitude poussa Bouvard, rapporteur, à proposer au jury de faire le vœu que l'exécution fût confiée à celui qui aurait le premier prix. Paul Planat, qui rapporte cet incident, souligne : "Il faut savoir gré à M. Salleron de cette protestation, surtout quand on n'est pas habitué à trouver chez les membres des jurys une vue aussi nette du devoir à accomplir $»^{6}$.

Le règlement sur les écoles publiques, publié en 1880, revient à l'enseignement simultané. Il précise que l'emplacement choisi pour l'école doit être sain, la construction solide. Les classes, rectangulaires à éclairage naturel venant à la gauche des élèves, comporteront au maximum 50 places. Les fenêtres seront rectangulaires, la hauteur sous plafond d'au 
moins 4 mètres. Il y aura un poêle par classe. Des préaux distincts pour les filles et les garçons. Un groupe complet ne pourra pas dépasser 750 élèves, 300 garçons, autant de filles et 150 pour la salle d'asile. Désormais Léon Salleron est considéré comme le véritable expert en matière de constructions scolaires. C'est comme tel qu'il approuve en 1887 le projet d'une École du livre qui deviendra l'École Estienne. On fait appel à lui pour visiter les chantiers en cours. Cependant on se préoccupe de diminuer le coût des écoles. En juillet 1889 le conseil municipal crée une commission « chargée d'étudier la manière d'éviter les projets dispendieux pour la construction d'écoles» Salleron en est le président, Bouvard, secrétaire. Il faut faire des économies, éviter la pierre de taille au profit de la pierre meulière pour les soubassements et les moellons pour les murs, remplacer, chaque fois que c'est possible les fosses d'aisance par le tout à l'égout. La commission élabore ses recommandations en matière de chauffage et d'aération. Le chauffage sera différent dans les pièces occupées de façon constante et celles qui ne le sont pas, comme les salles à manger, dortoirs ou les chambres à coucher ${ }^{7}$. On assiste alors à la construction de nombreux groupes scolaires par les architectes de la Ville. En effet ce sont les architectes du service permanent qui construisent les écoles. Le service temporaire fonctionne grâce à eux. Ce tour de passe-passe maintient la fiction d'un service temporaire indépendant pour éviter les critiques de la profession, qui pourrait se plaindre d'être évincée des chantiers. En fait le service permanent fonctionne comme une véritable agence communale d'architecture ${ }^{8}$.

\section{Les travaux officiels}

\section{L'Opéra de Paris}

13 À la fin de l'année 1860, Walewski, le ministre d'État, met au concours le projet d'un grand opéra pour Paris. On demande seulement une esquisse, le programme étant très succinct et les délais très courts. Léon Salleron figure parmi les 171 candidats dont les projets sont exposés au Palais de l'industrie. Les concurrents devaient produire un plan d'ensemble, une élévation géométrale de la façade principale, une coupe sur la longueur de la salle, un devis sommaire descriptif et estimatif. Salleron n'est pas au nombre des cinq prix décernés. Au terme d'un deuxième concours, c'est Garnier qui est retenu. Le projet de Salleron est conservé dans les collections de l'Académie d'architecture ${ }^{9}$. Il figure parmi les dix-huit projets publiés par la Revue générale d'architecture. ${ }^{10}$

\section{Les écoles}

De 1870 à sa retraite en 1879 Léon Salleron ne construit ou n'aménage pas moins de vingtsix bâtiments scolaires. Sur les dix écoles, dont la construction est décidée en 1873, il est chargé du groupe scolaire de la rue Riblette, dans le $\mathrm{XX}^{\mathrm{e}}$ arrondissement. En 1876, sur les douze autres prévues, il est chargé de trois nouvelles écoles. Il est apprécié, car il ne dépasse jamais les crédits alloués et reste toujours un peu en dessous ${ }^{11}$. Tous les plans aquarellés de ces écoles sont conservés au Archives de Paris, mais dans un tel état de fragilité, qu'ils sont parfois impossibles à consulter. Nous en conservons des 
reproductions grâce à l'ouvrage de Narjoux sur les bâtiments publics construits de 1850 à $1880^{12}$.

Parmi ses principales constructions scolaires il faut citer :

- le groupe scolaire de la rue Riblette, $\mathrm{XX}^{\mathrm{e}}$, construit de 1874 à 1876, destiné à recevoir 650 élèves pour un coût total de 442000 francs Les classes comportent de 80 à 60 élèves ce qui est supérieur aux normes qui seront définies en 1880 .

- l'école de garçons et de filles, 9 rue Blanche, IX ${ }^{e}$, construite de 1878 à 1880 pour 390500 francs

- l'école primaire du 2-4 rue Fessart, XXe, construite en 1876-1880 pour 549884 francs

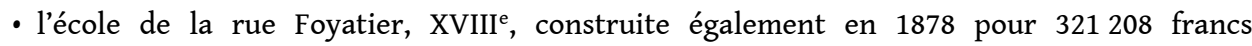
aujourd'hui détruite.

- l'école de garçons, 16 rue Julien Lacroix, XXe, construite en 876-1878, reconstruite en 1879 pour 208065 francs

- le groupe scolaire du 75 bd de Belleville, $\mathrm{XI}^{\mathrm{e}}$, construit d'avril 1877 à juin 1878 pour 660840 francs L'école de fille et l'école de garçons contiennent chacune 350 places, l'asile 220. Le groupe renferme en outre une école de dessin pour 180 élèves.

- l'asile rue du Jourdain, $\mathrm{XX}^{\mathrm{e}}$, construit de 1876 à 1878 pour 150142 francs

- l'école normale d'instituteurs de la Seine, rue Molitor, $\mathrm{XVI}^{\mathrm{e}}$, construite de 1878 à 1882 pour 120 élèves pour un coût de 1500000 francs l'établissement comprenait 3 classes, un amphithéâtre de physique, une bibliothèque, deux salles de dessin, une salle de chant, une salle d'armes et un atelier d'ouvrages manuels. Les élèves ont des chambres indépendantes.

Dans l'ensemble ces constructions sont assez simples, sans apprêt et à faible coût. Leur architecture reflète parfaitement leur fonction. Elles se rapprochent en cela de l'école rationaliste illustrée par Labrouste et Vaudremer. Les plus anciennes ne répondent pas aux normes de 1880, avec, en particulier, un nombre élevé d'élèves par classe. Plusieurs de ces réalisations sont néanmoins remarquables, en particulier l'École normale d'instituteurs et le groupe scolaire du boulevard de Belleville dont les plans ont été exposés à l'exposition universelle de Paris en 1878 et pour lequel Salleron a obtenu une médaille, avec ses deux parties symétriques, et ses imposantes façades, ornées par la suite de médaillons de grands hommes. Ces constructions ont été plusieurs fois reproduites dans les revues d'architecture de l'époque.

D'une façon surprenante, l'architecte Anne-Marie Châtelet, auteur de plusieurs ouvrages sur les constructions scolaires, ne cite que rapidement Léon Salleron, en lui attribuant un prénom erroné ou en le confondant avec son neveu René et ne publie aucun de ses plans alors que dans ses Éléments et théorie de l'architecture, repris de ses cours à l'École des beaux-arts, Julien Guadet se réfère explicitement aux travaux de Salleron. 
Groupe scolaire de Belleville.

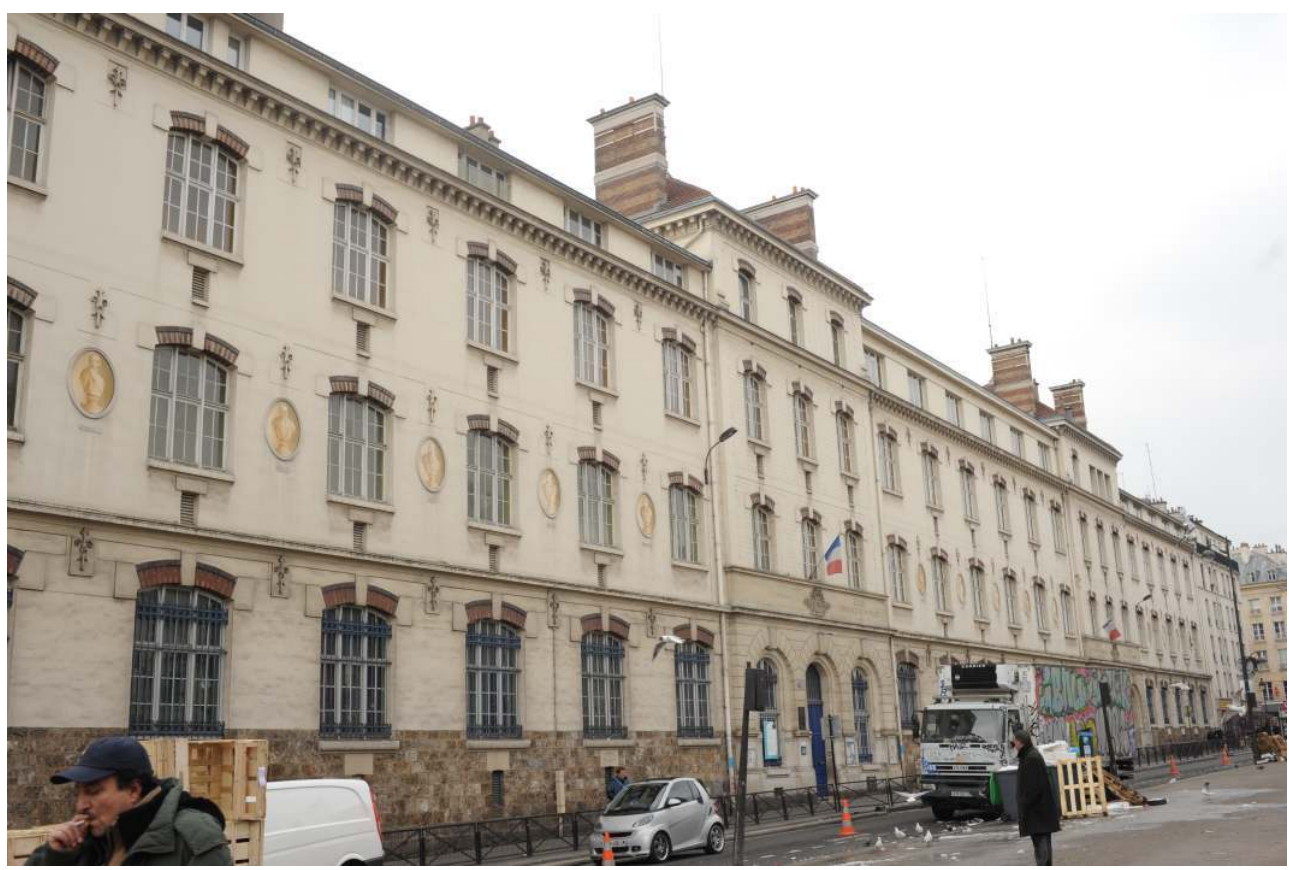

Un des pavillons centraux

Cl. Daniel Maunoury

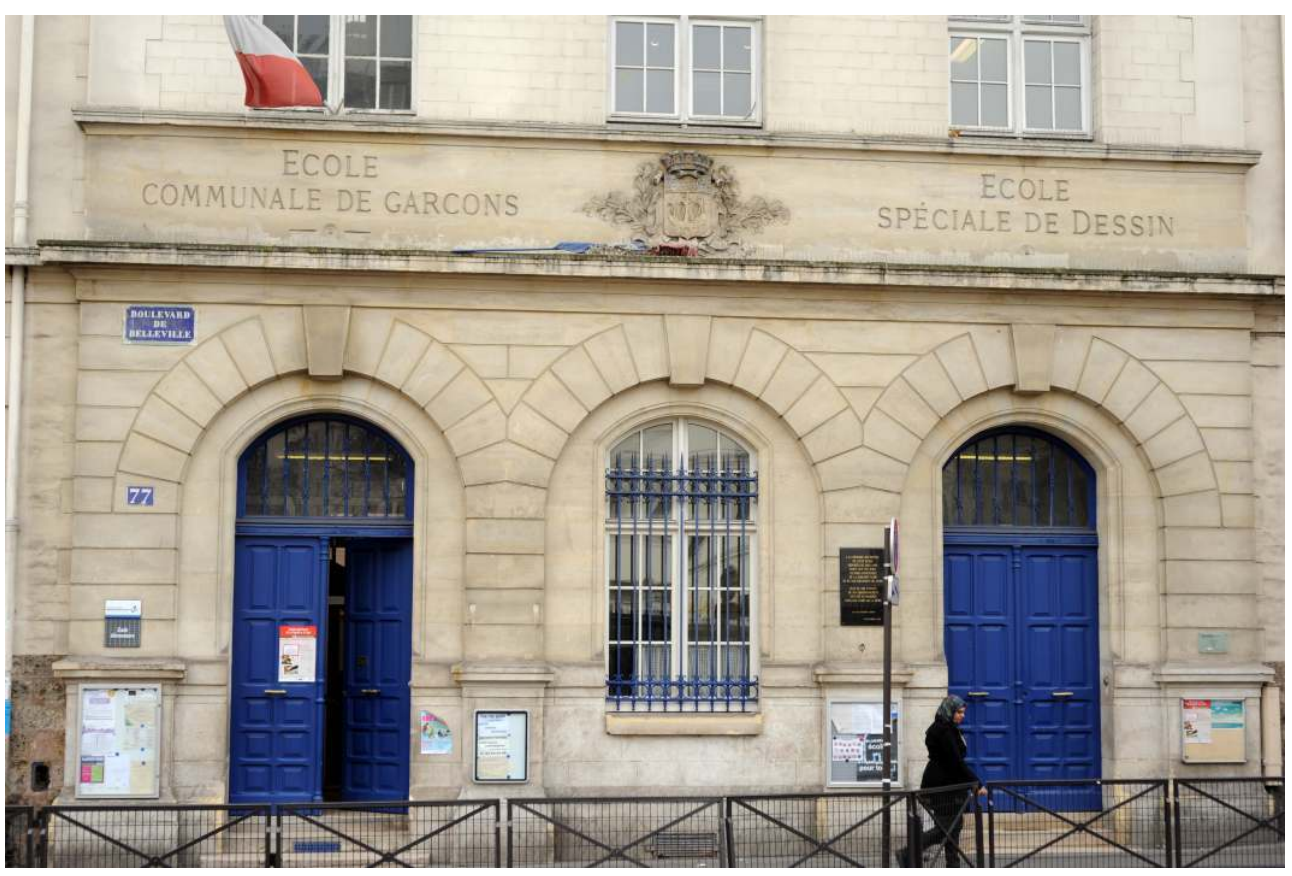

Façade.

Cl. Daniel Maunoury

\section{La mairie du XXe arrondissement}

18 Une des réalisations les plus connues de Léon Salleron est la mairie du $\mathrm{XX}^{\mathrm{e}}$ arrondissement. De nombreux ouvrages lui ont été consacrés et il faut mentionner ici le travail de M. Thierry Halay, président de la Société historique du XX ${ }^{\mathrm{e} 13}$. 
19 Après 1860 et l'annexion des communes périphériques, la rue de Belleville devint la frontière entre les nouveaux $\mathrm{XIX}^{\mathrm{e}}$ et $\mathrm{XX}^{\mathrm{e}}$ arrondissements. La mairie du $\mathrm{XX}^{\mathrm{e}}$ arrondissement, d'abord logée dans les bâtiments de l'ancienne mairie de Belleville, l'ancienne guinguette de "l'île d'amour », fonctionna ainsi pendant trente ans dans des locaux particulièrement incommodes. Il fallait construire un bâtiment plus grand et mieux adapté à une population en très forte augmentation. On chercha un endroit plus central. Ce fut en bordure d'une place circulaire nouvellement créée, dénommée place du Pueblo, puis des Pyrénées, et enfin place Gambetta depuis 1893, à l'intersection des actuelles avenues Gambetta et Belgrand.

Les travaux commencèrent en 1867 et furent plusieurs fois retardés. En 1870 il fallut faire des travaux considérables pour consolider le sous-sol. Dans ses mémoires Haussmann souligne que la nature glaiseuse du sol nécessita la construction d'une vaste plateforme de béton sur laquelle reposent les fondations profondes de 19 mètres. Pendant les événements de 1870-1871 les travaux furent interrompus. En 1873 ils n'en étaient qu'au rez-de-chaussée et avaient déjà coûté 564000 francs. Ils n'ont repris qu'après une pétition des habitants au Préfet de la Seine. Le Conseil de Paris vote alors un crédit de 626736 francs pour l'achèvement des travaux. Léon Salleron confie les sculptures de la façade à Legrain et les ornements intérieurs à Watrinelle et Germain. Un crédit de 12440 francs est consacré aux trois sculpteurs. Les plans ont dû être revus à la baisse à cause du surcoût provoqué par la consolidation du terrain. Le coût définitif, considérable, est évalué par Haussmann à 1450000 francs, chiffre également donné par Narjoux, alors qu'en 1872 la Gazette des architectes prévoyait un montant de 1980000 francs. Pour cette importante commande Salleron ne ménagea pas sa peine, près de 40 plans et dessins de sa main sont encore conservés au cabinet des arts graphiques du Musée Carnavalet, témoins des différentes étapes du projet ${ }^{14}$. Sans vouloir décrire ici en détail un bâtiment bien connu, rappelons que l'architecte a prévu une façade assez imposante sur le petit côté du trapèze occupé par la mairie, avec trois grands porches en plein cintre, surmontés à l'étage supérieur par trois fenêtres à meneaux; l'attique est surmontée d'un campanile. De part et d'autre de la façade deux pavillons mènent à des ailes latérales de moindre hauteur. L'ensemble rappelle le style Renaissance, parti typique de l'atelier Duban, ce qui a fait classer Salleron par Hautecoeur parmi les architectes éclectiques.

21 Comme pour les bâtiments scolaires, la construction des mairies était précisément encadrée et cette mairie présente des parentés avec celles construites à la même époque et sur des emplacements similaires, en particulier la mairie du XI construite de 1862 à 1865 par Gancel et surtout la mairie du XIII ${ }^{e}$, construite sur la place d'Italie de 1873 à 1877 par Paul Bonnet. On y retrouve les trois porches en plein cintre surmontés de trois baies et le campanile sur le toit. La mairie du $\mathrm{XX}^{\mathrm{e}}$ se caractérise par sa simplicité et son caractère monumental accentué par la présence des deux pavillons en retrait.

Signalons en passant que Salleron participa à l'aménagement de la mairie du XVIII ${ }^{e}$, œuvre de Marcellin Emmanuel Vercollier, élève de Baltard. C'est lui qui construisit la grande salle des fêtes.

23 Les plans de la Mairie du XXe ont été exposés par la Ville de Paris à l'exposition universelle de Vienne en 1873 et à celle de Londres en 1875. Léon Salleron y récolta des médailles. À l'exposition universelle de Paris, en 1878 ont été exposés les plans, coupes et façades de la mairie du XX ${ }^{e}$, de l'asile de la rue du Jourdain et des groupes scolaires de la rue Riblette et du boulevard de Belleville. Figurait également un modèle réduit à l'échelle de $2 \mathrm{~cm}$ pour $1 \mathrm{~m}$ du groupe scolaire de Belleville, qui a disparu. Léon Salleron y fut 
récompensé par une deuxième médaille, ex-aequo avec les architectes de la Ville de Paris. Vaudremer, auteur du rapport sur l'exposition conclut enthousiaste : "Tout en rendant aux exposants étrangers le juste tribu d'éloges qui leur est dû, il n'est douteux pour personne qu'à l'exposition de 1878, comme dans les expositions antérieures, la supériorité est incontestablement acquise aux architectes français quant à l'entente générale de la composition. De tous points on vient étudier à l'École des beaux-arts de Paris [...] On ne peut se dissimuler que les efforts, les idées, les inspirations sont dominés depuis longtemps par la philosophie éclectique qui a envahi notre société tout entière ${ }^{15}$. »

\section{Les constructions privées}

\section{Hôtels particuliers et maisons à loyers}

Si Léon Salleron est surtout connu et cité pour la mairie du $\mathrm{XX}^{\mathrm{e}}$ et les nombreuses écoles qui ont fait sa réputation, il a construit également pour des particuliers qui participent à l'élan bâtisseur de cette époque. Sans doute, outre le revenu financier supplémentaire que cela lui procure, apprécie-t-il de s'évader du carcan des commandes officielles.

Plusieurs catégories de bâtiments coexistent alors. Tout d'abord les hôtels particuliers, comme au siècle passé, construits pour une seule famille, le plus souvent dans des quartiers neufs, qui vont devenir rapidement à la mode, puis les maisons à loyers, dans lesquelles chaque étage comporte alors un ou plusieurs appartements, qui apparaissent également dans les beaux quartiers pour une clientèle riche, qui veut faire des placements; enfin des immeubles de rapport plus modestes. Tous ces bâtiments respectent la grammaire des immeubles haussmanniens: façade en pierre de taille sans saillie, balcons aux second et cinquième étages, en fer forgé ou en fonte de fer. Ils ne se distinguent que par des ornements souvent discrets.

Bien que comme spécialiste des constructions scolaires, Léon Salleron ait été appelé à faire partie de nombreux jurys en province, sa clientèle privée provient exclusivement de la région parisienne, y compris pour ses réalisations de ce qu'on n'appelait pas encore des résidences secondaires, châteaux et hôtels particuliers en province pour des membres de la grande bourgeoisie.

La première construction privée de Léon Salleron est le grand immeuble du 61 boulevard Malesherbes, qui date de $1861^{16}$. S'élevant sur quatre étages, plus des combles, elle est surtout remarquable par sa décoration avec les médaillons des quatre saisons, dus à Aimé Millet, qui ornent le premier étage. Nous n'en avons pas le permis de construire, la série ne débutant qu'en 1882 aux Archives de Paris. Cet immeuble existe toujours ${ }^{17}$. 


\section{1 boulevard Malesherbes}

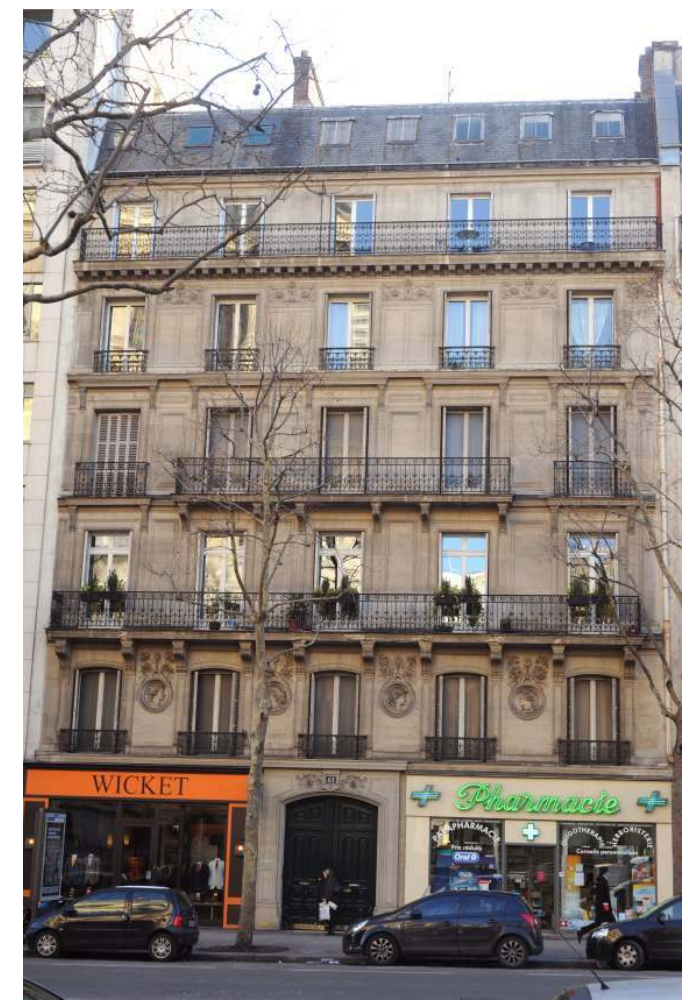

Façade.

Cl. Daniel Maunoury

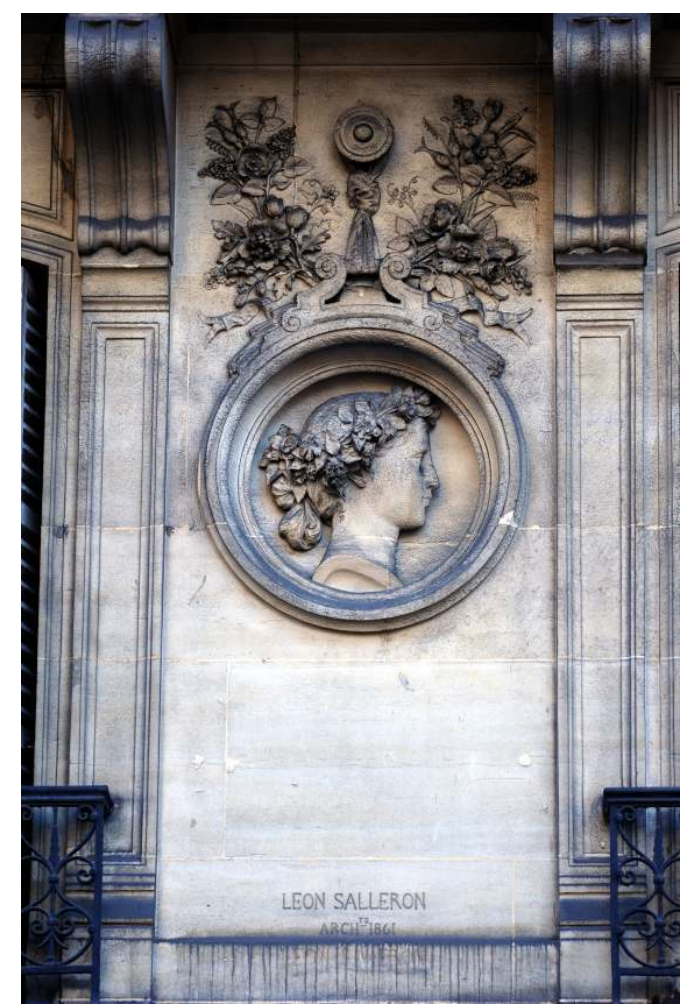

Médaillon avec signature

Cl. Daniel Maunoury 
L'année 1878, dernière année avant sa retraite, est particulièrement fertile en réalisations. Tout en réalisant de nombreuses écoles, Salleron dépose plusieurs permis de construire; tout d'abord pour lui-même et sa famille: une maison de rapport sur un terrain qui lui appartient au 15 rue Portalis, VIII ${ }^{e}$. C'est là qu'il installera son agence personnelle par la suite. Pour l'heure, il demeure toujours 40 rue de Rome. Il construit un hôtel particulier au 17 de la même rue pour son gendre Homberg qui a épousé sa fille Thérèse. Dans le $\mathrm{XVI}^{\mathrm{e}}$ arrondissement, rue Saint-Didier, il bâtit deux hôtels particuliers aux 45 et 49 pour M. Villebessex deux autres hôtels aux 51 et 53 pour E. de Malaincourt. Dans le $1^{\text {er }}$, à l'angle des rues de Rivoli et des Feuillants, une maison de rapport pour Lefevre de la Vieuxville. Mais c'est après sa retraite que ses constructions privées sont les plus nombreuses. Certaines d'entre elles ne nous sont connues que par La Semaine des constructeurs, publiée par César Daly et Paul Planat, qui recense chaque semaine les travaux privés qui se font à Paris, d'autres ont fait l'objet de descriptions détaillées dans La Construction moderne, revue dirigée par Planat. La qualité exclusivement parisienne des sources disponibles fausse probablement les perspectives.

On peut mentionner :

- 1879, 69 avenue de la Grande-Armée, construction d'un immeuble de rapport pour Filon ;

- 1880, 4 rue Linné, un immeuble de rapport pour le parfumeur P. Pinta, avenue de l'Opéra. Cet immeuble existe toujours et reste propriété de la famille.

- 1881 , boulevard Suchet, construction pour la famille Duboys d'Angers ${ }^{18}$.

- 1884 le grand immeuble de rapport Romaguera abondamment décrit par la Construction moderne de 1887/1888 ${ }^{19}$. Bien que donné pour disparu par Roland Pozzo di Borgo dans son ouvrage sur les Champs-Elysées, cet immeuble existe toujours, occupé maintenant par le Crédit lyonnais. On peut toujours y voir la signature L. Salleron et Homberg 1886. Lors de la construction, il comporte quatre étages carrés et comble mansardé, il a deux appartements à chaque étage, les combles sont occupés par les domestiques et les locataires des boutiques du rez-de-chaussée sur l'avenue. Le rez-de-chaussée sur la rue Pierre Charon est occupé par un appartement.

- 1886 plusieurs immeubles, un immeuble de rapport au 18 avenue Hoche pour sa cousine Victoire Lucile Marcellot, veuve Gentil ;

deux immeubles à loyer, l'un au 42-43 rue de Paradis pour Pinet, l'autre au 88 rue de Monceau à l'angle de la rue du Général- Foy pour Coutelet, également décrit dans la Construction moderne $e^{20}$.

- 1889 un hôtel particulier pour sa cousine Gentil au 77 rue du Faubourg Saint-Honoré. Cet hôtel, qui existe toujours, est occupé actuellement par la galerie Steinitz. 
Hôtel particulier

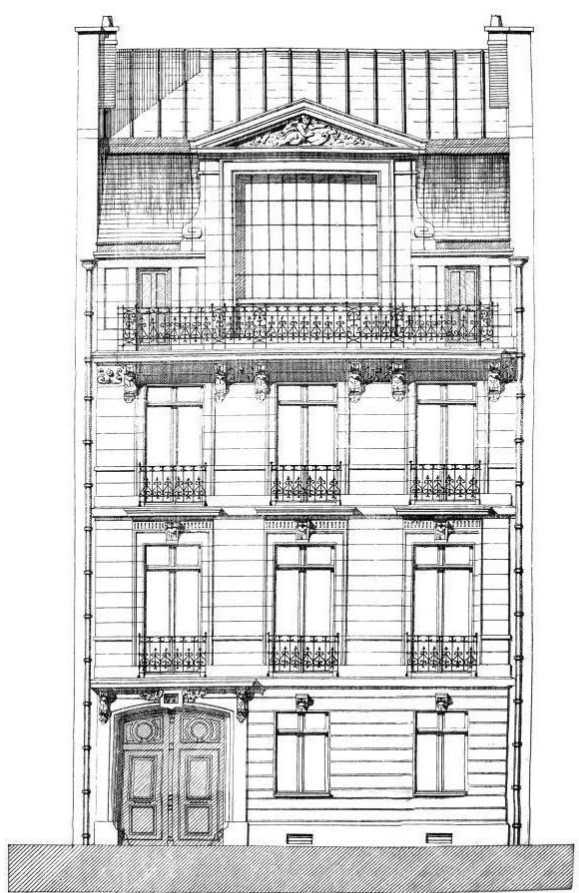

77 rue du Faubourg Saint-Honoré, VIII arr. Projet.

Cl. Daniel Maunoury 
Hôtel particulier

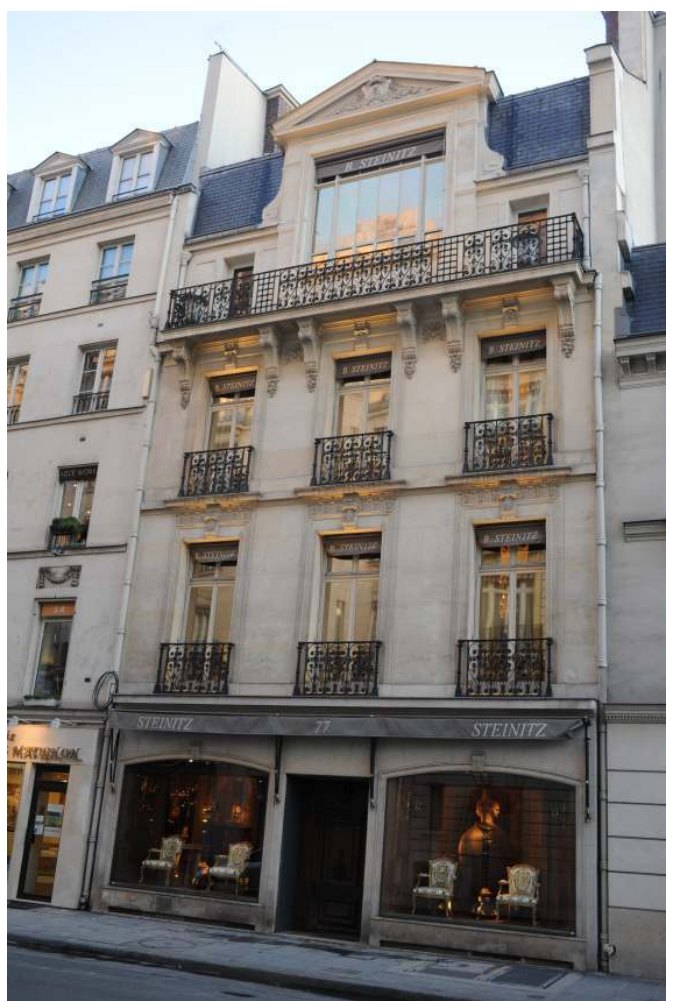

77 rue du Faubourg Saint-Honoré, Galerie Steinitz, VIII arr.

Cl. Daniel Maunoury

- 1892 un immeuble de rapport 18 rue Cuvier, qui est encore propriété de la famille Pinta aujourd'hui, ainsi qu'un autre immeuble 14 rue de Logelbach pour Lorrain.

- 1897 un immeuble à loyer au 121 avenue des Champs-Élysées pour Carraby, aujourd'hui démoli. 
Immeuble Romaguera

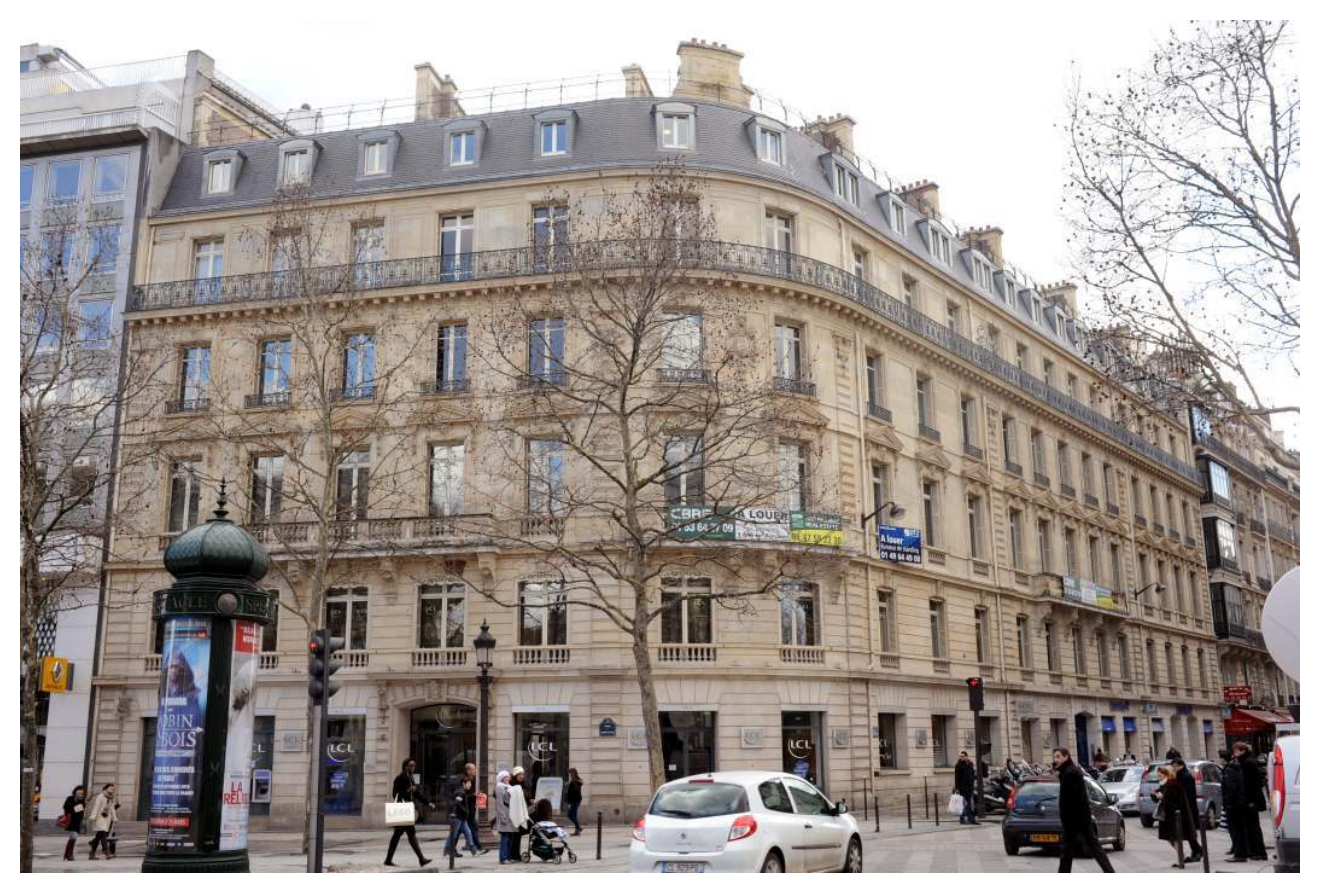

55 avenue des Champs-Élysées, VIII arr.

$\mathrm{Cl}$. Daniel Maunoury

\section{Série d'hôtels particuliers}

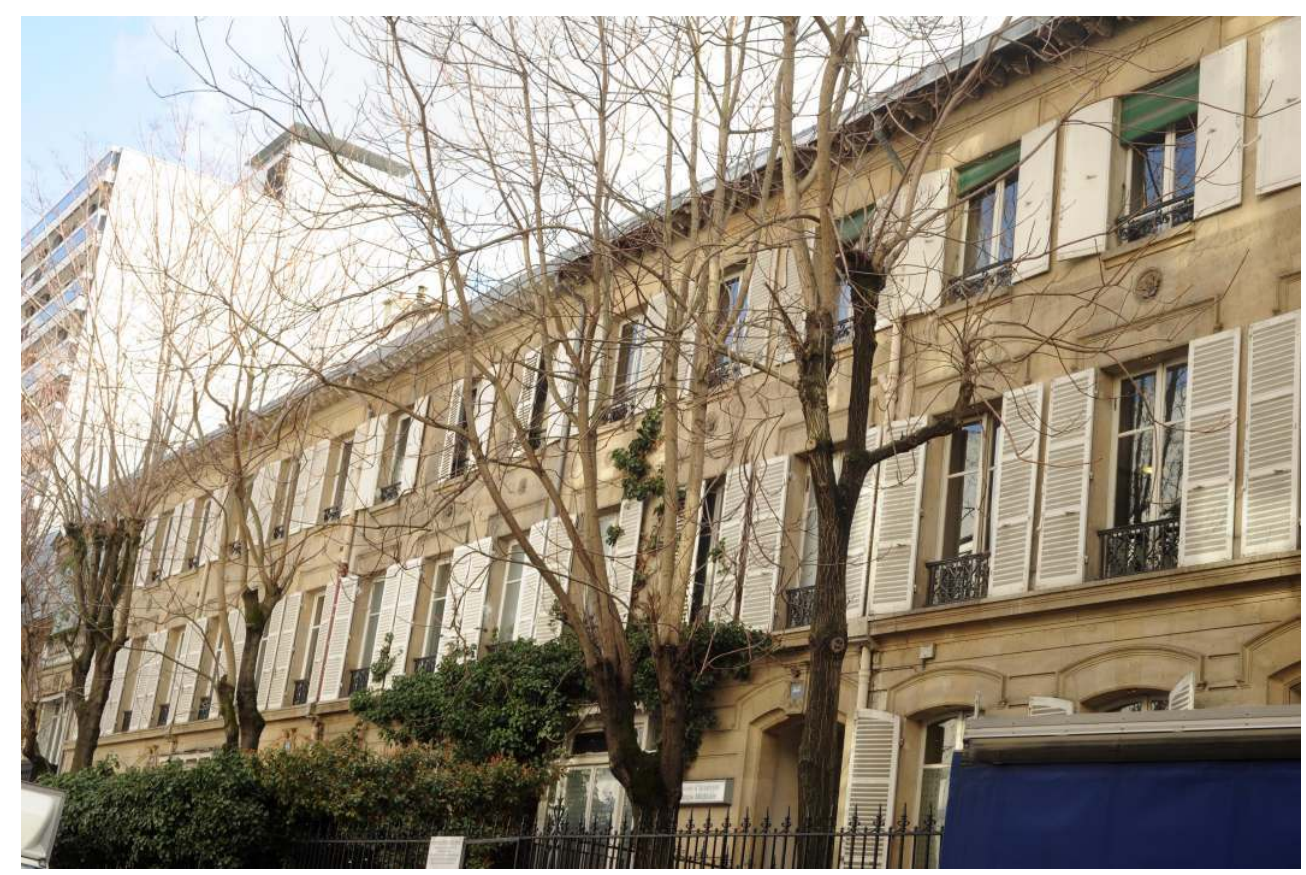

49-55 rue Saint-Didier, XVIe arr.

$\mathrm{Cl}$. Daniel Maunoury

Un hôtel particulier construit avenue Raphaël pour lequel nous n'avons ni la date, ni le nom du propriétaire a été minutieusement décrit par Planat ${ }^{21}$. On peut raisonnablement l'identifier en réalité avec l'hôtel Duboys d'Angers du boulevard Suchet, les immeubles 
des premiers numéros ayant des entrées dans ces deux voies. Construit pour une personne âgée et infirme, toutes les pièces principales sont au même niveau: salon, chambre à coucher avec une chambre de domestique attenante, salle de bains et watercloset ; la cuisine et les dépendances sont au sous-sol ; l'étage mansardé comprend deux chambres d'amis et des chambres de domestiques. L'ensemble a coûté 179200 francs auxquels il faut ajouter 20000 francs pour les écuries et remises construites par la suite.

Dans son discours funèbre, Bertaumieux fait état de plus de quarante maisons de rapport.

En dehors de Paris, Léon Salleron construisit de nombreuses villas à Versailles et en Seine-et-Oise, dont il est difficile de trouver la trace, faute de permis de construire. Le dépouillement des registres de l'étude CXVII, $\mathrm{M}^{\mathrm{es}}$ Daguin, puis Delapalme, notaires habituels de la famille, pour les années 1856-1890 n'a pas permis de retrouver de contrat avec des propriétaires.

\section{Villa d'Élisabeth Joubert}

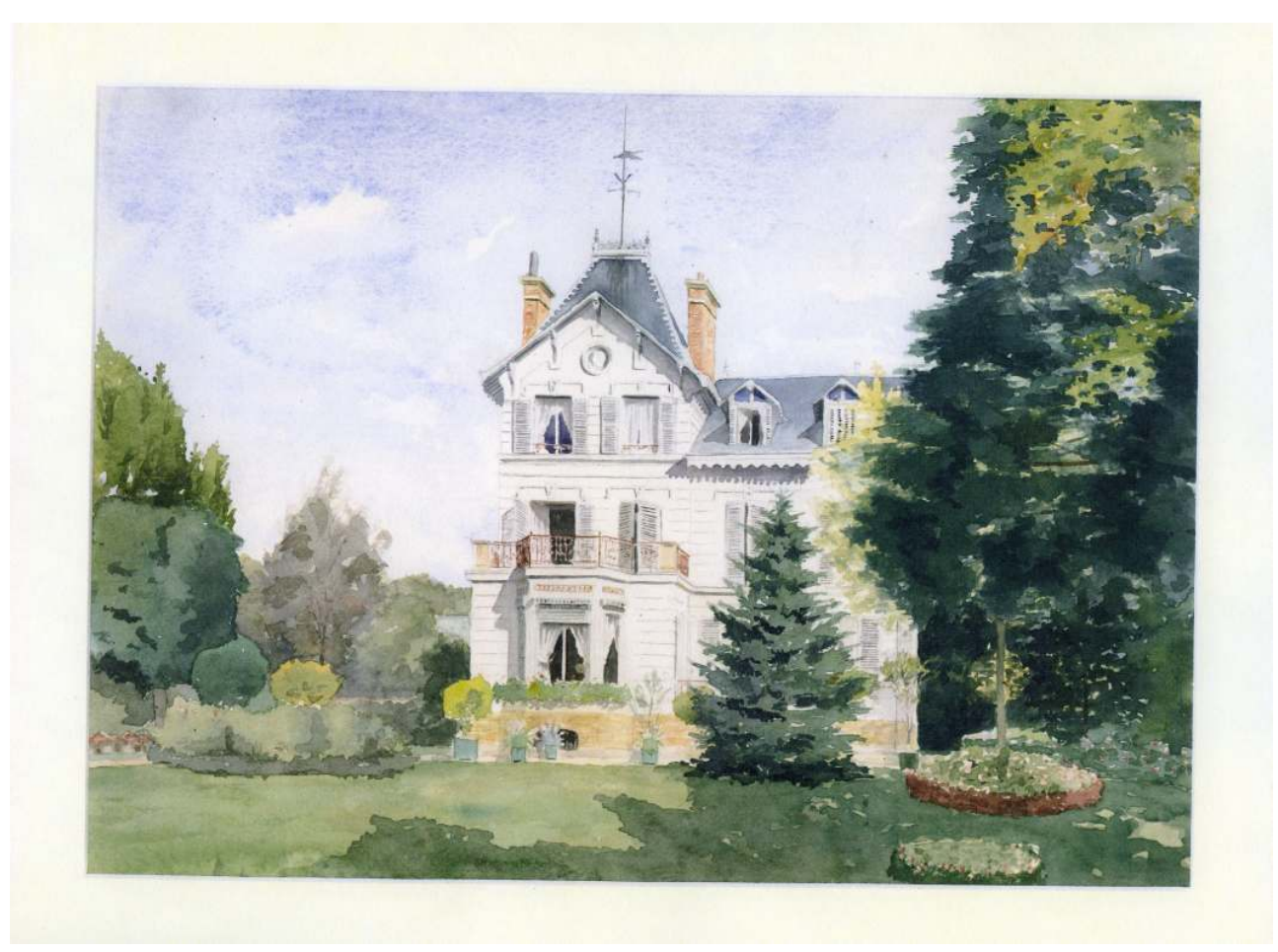

Rue de Montebello à Versailles (Yvelines), aquarelle de 1883 de René Salleron.

Coll. part.

Salleron fut également l'architecte de constructions plus ambitieuses tels le château de Saint-Voir, dans l'Allier, construit dans le style Louis XIII ${ }^{22}$ et du château de Blanchelande à Montmerré, dans l'Orne pour Eugène Poriquet, doyen d'âge du Sénat à l'occasion du mariage de ce dernier avec Anne-Clémence Colin, issue peut-être d'une famille alliée à la famille Salleron. Ce château, achevé en 1870, construit en briques et granit de style Louis XIII, présentait de grandes similitudes avec Saint-Voir. Il a aujourd'hui disparu. Une autre de ses réalisations est la construction d'un superbe hôtel particulier «sur le modèle des hôtels de l'avenue du Bois-de-Boulogne ", faubourg Saint-Roch, à Romorantin, Loir-etCher, pour Aristide Normant, industriel propriétaire d'une grande usine de draperie ${ }^{23}$. Cet hôtel, dont la construction s'échelonne de 1874 à 1877 est d'un grand confort. Son aménagement reflète le niveau de vie d'un riche propriétaire. Il comprenait deux étages, 
sur un sous-sol occupé par des caves, une laverie, un séchoir, la cuisine avec la salle à manger des domestiques, une salle d'armes et l'emplacement du calorifère. Au rez-dechaussée, vestibule, office, salle à manger, salon, salle de billard, petit salon, bureau, chambre à coucher du gardien, cabinet de toilette et serre. Au premier étage se trouvent cinq chambres à coucher, cabinets de toilette, water-closets. Le deuxième étage est occupé par des chambres supplémentaires, les chambres des domestiques, lingerie et water-closets. Après la mort de son propriétaire, décédé le 14 avril 1889 sans enfants, l'hôtel, estimé à 83228 francs, est mis en adjudication au prix de 40000 francs Il fut acheté par la suite par la Ville de Romorantin en 1952 et sert aujourd'hui d'Hôtel de ville.

\section{Château de Saint-Voir (Allier)}

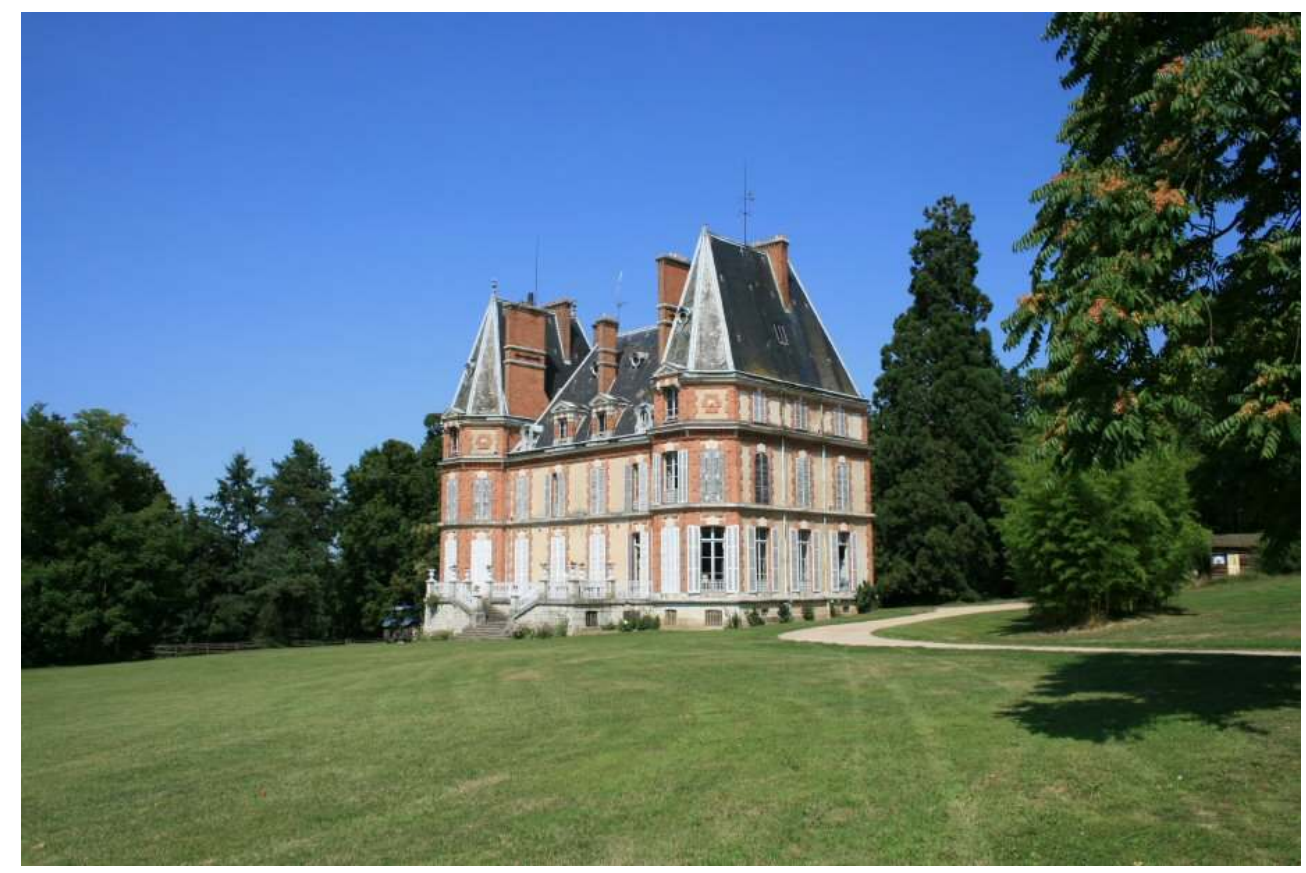

Cl. Delageneste 


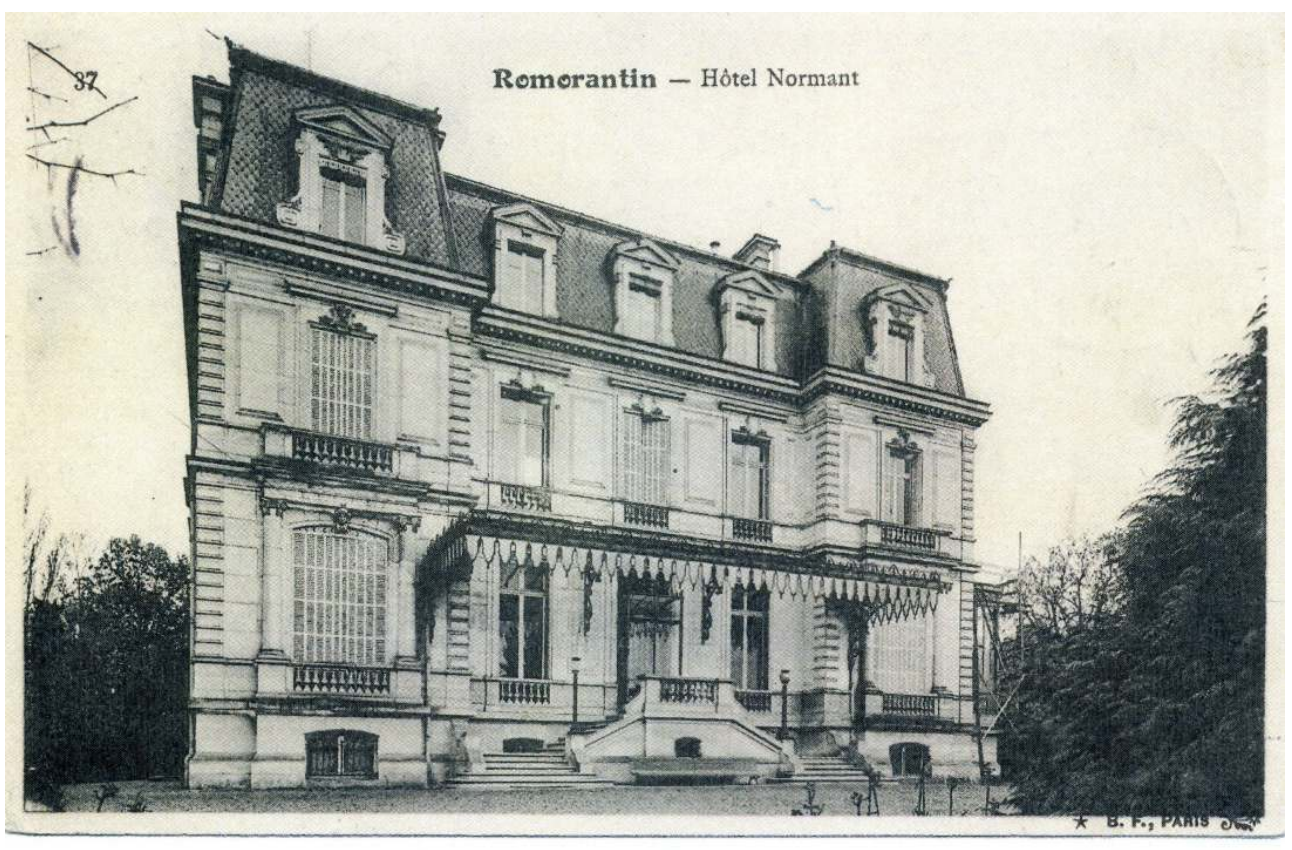

Carte postale

Archives départementales du Loir-et-Cher, 6Fi 194/157

David de Penanrun et Delaire citent en outre d'autres hôtels ainsi que des couvents mais, faute de savoir leur localisation, il n'a pas été possible de les retrouver. Léon Salleron est aussi l'architecte de tombeaux au cimetière de l'Est, aujourd'hui du Père-Lachaise, pour les familles Neron et Boutet. Daly fait figurer ces tombeaux sous la rubrique « la foi ». Le tombeau de la famille Boutet a la forme d'une petite chapelle, surmontée d'une croix.

Comme son cousin Jules-Bernard, fabricant d'instruments de précision, Jules Salleron est également ingénieur. On lui doit la conception d'un appareil pour le filtrage des eaux, appareil solide et rustique permettant un filtrage assez rapide ${ }^{24}$. C'est probablement à ce titre qu'il est nommé en 1898 membre de la commission chargée d'étudier et de formuler les mesures qu'il convient de recommander aux propriétaires pour assurer, à l'intérieur de leurs immeubles, l'application de la loi du 10 juillet 1894 et de l'arrêté du 24 décembre 1897 sur l'écoulement direct à l'égout des matières de vidange..$^{25}$

Pour ses constructions privées La Société centrale des architectes lui décerna en 1880 la grande médaille pour travaux d'architecture privée.

\section{Constructions religieuses}

Les constructions religieuses de Léon Salleron sont rares, les commandes publiques étaient le domaine réservé de Vaudremer. On ne voit apparaître sa signature que pour des chapelles modestes d'origine privée.

La seule commande officielle, date du temps où il était en charge du XXe arrondissement, encore ne put-il la mener à bien, par suite les atermoiements du Conseil municipal. Il s'agit du temple de la rue Julien Lacroi ${ }^{26}$, réclamé par la communauté protestante de Belleville, pour lequel la municipalité avait acquis un terrain en 1864. Dès l'année suivante Salleron soumet un projet, suivi d'un second en 1867. En 1870 le conseil 
presbytéral approuve le projet d'un temple de 576 places, qui est stoppé par la guerre et les événements. En 1874 un projet plus restreint et moins coûteux est de nouveau soumis au Conseil municipal, qui cependant ne l'adopte toujours pas. Il faut une pétition des habitants de Belleville, un rapport du président du consistoire et un autre du directeur de l'administration municipale pour que le Conseil municipal adopte finalement le projet en avril 1876. C'est alors Vaudremer, nouvellement nommé responsable des bâtiments religieux, qui en est chargé. Il reprit d'ailleurs les plans tracés par Salleron et la façade, fort simple est celle que ce dernier avait conçue. La construction sera achevée en 1881.

La chapelle de Clagny, à Versailles, est un projet plus personnel. Construite grâce à une souscription comme chapelle privée à l'angle des rues du Parc-de-Clagny et de Villeneuve-l'Étang, où Léon Salleron se construira une villa, elle est l'œuvre de ce dernier, qui abandonne pour ce projet ses honoraires d'architecte. Nous possédons un exemplaire de la lettre circulaire, datée d'août 1866, appelant à une deuxième souscription pour compléter les fonds déjà recueillis ${ }^{27}$. Elle est signée Gauthier, propriétaire et Salleron, architecte. Les premiers souscripteurs, au nombre desquels figurent de nombreux membres de la famille, outre Léon Salleron lui-même, sa sœur, sa belle-sœur, sa bellemère, une de ses cousines germaines, avait déjà recueilli 23370 francs sur les $29000 \mathrm{fr}$. représentant le coût total du terrain et de la construction. C'est donc une construction assez modeste, en pierres meulières, avec un petit porche surmonté d'un campanile, de style italien. D'après la lettre de quête, elle réunissait environ 150 personnes chaque dimanche en 1866. Elle est aujourd'hui désaffectée, a été entresolée et sert de salle paroissiale.

\section{Chapelle de Clagny}

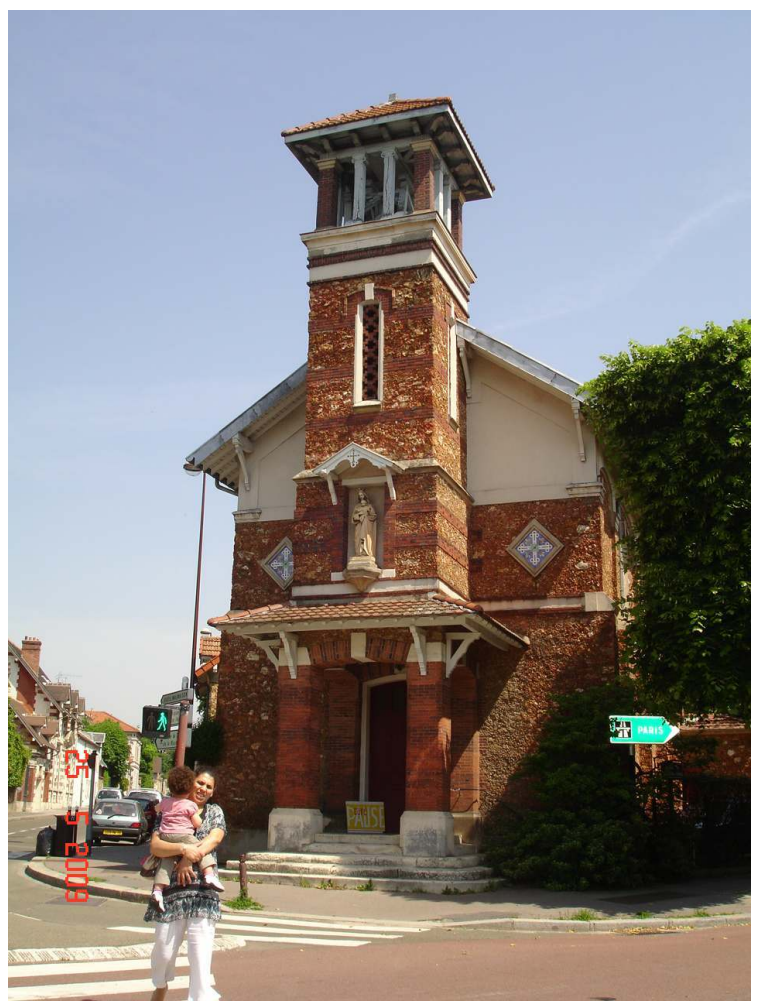

Rue du Parc-de-Clagny, Versailles (Yvelines). Porche et bas-côté

$\mathrm{Cl}$. de l'auteur 


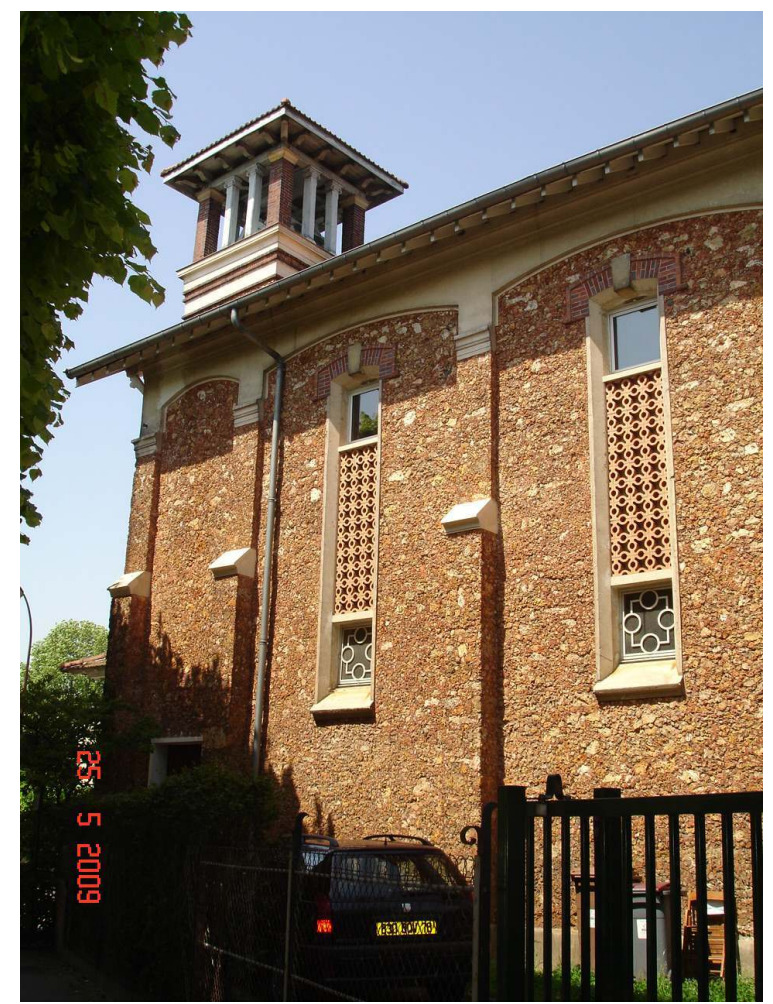

Rue du Parc-de-Clagny, Versailles (Yvelines). Bas-côté.

Cl. de l'auteur

Deux autres réalisations également modestes peuvent être signalées : la chapelle de l'Ave Maria, du monastère des Clarisses, 5 villa de Saxe, dans le $7^{\mathrm{e}}$ arrondissement, construite en 1883-1884 et celle du Collège Gerson, 31 rue de la Pompe, dans le $16^{\mathrm{e}}$, construite en 1895, l'une et l'autre en collaboration avec Eugène Homberg. La chapelle de l'Ave Maria est constituée d'une nef en deux parties séparées par le maître-autel. La plus grande est couverte d'une voûte d'arrêtes et possède des fenêtres cintrées, l'autre est couverte d'un plafond. La chapelle du Collège Gerson se compose d'une simple nef à chevet plat couverte d'une charpente apparente. Le sol est dallé de mosaïques dont le semis de lauburu rappelle le souvenir du fondateur du collège, l'abbé Dibildos, d'origine basque.

\section{Élèves, collaborateurs et collègues}

41 Salleron n'a jamais eu, comme Vaudremer, Pascal et bien d'autres, d'écurie de jeunes élèves. Outre son neveu, René Salleron (1850-1943), qu'il patronna dans l'existence, David de Penanrun ne lui connaît que cinq élèves, dont aucun n'a été célèbre. Il s'agit d'HenriAlexandre Bernard, né en 1849 à Mondidier, qui devint inspecteur des monuments historiques et fut architecte à Compiègne ; Louis Brunet, né en 1869 à Versailles, devenu architecte à Versailles; Victor, Marie, Ferdinand Genay, né à Nancy en 1846, architecte des hospices et hôpitaux civils de Nancy, qui termina sa carrière comme inspecteur des édifices diocésains de Nancy ; Jules, Romuald, Perdican Rischmann, né en 1844 à Paris, architecte du ministère des finances, rue de Rivoli, qui exerça à Bois-Colombes ; Gustave Sirois, né en 1840 à Paris, pour lequel on ne dispose d'aucun autre renseignement. 

ses équipes. On peut citer Albert Béziers-Lafosse, né en 1840, qui travailla à l'agence de la caserne de l'octroi au bastion 10 ; Joseph-Antoine Bouvard, inspecteur pour la mairie du $\mathrm{XX}^{\mathrm{e}}$, qui devint directeur administratif des services d'architecture et des promenades et des plantations de la Ville et à qui Léon Salleron recommanda son neveu René ; Eugène Théodore Homberg (1846-1925), qui travailla à l'agence du groupe scolaire de la rue Blanche et qui épousa Thérèse Salleron ; Louis-Étienne Laisné (1834-1886) ; Jean-Baptiste, Jules Albert Leclerc, né en 1840, fut son inspecteur pour la mairie du $\mathrm{XX}^{\mathrm{e}}$ et l'École normale d'Auteuil; Marie, Auguste Naudet, né en 1849 à Paris; Eugène Quellain, inspecteur pour les écoles des rues Fessard et Palestine ; Georges, Alexandre Roger, né en 1834, fils de Napoléon, Alexandre, Jules; Eugène Suffit (1831-1895), qui travailla à l'École normale d'instituteurs.

43 À la Ville de Paris et comme expert en constructions scolaires, Salleron côtoya de nombreux architectes aujourd'hui connus Narjoux, Train, Trélat, Vaudremer et Viollet-leDuc.

Bon collègue, on le trouve parmi les signataires de la pétition remise au Président de la République par Delaunay, Trélat et Vaudremer pour protester contre la révocation de Coquart, architecte de la Cour de cassation et de l'École des Beaux-arts, révoqué en 1890 par le ministre des travaux publics pour avoir fait traîner les travaux de la Cour de cassation et avoir dépensé les crédits sans les terminer ${ }^{28}$.

Membre du Conseil d'architecture du département de la Seine, Léon Salleron restait cependant attentif aux ouvriers qui réalisent les projets des architectes. Il créa en 1882 une fondation à l'Académie d'architecture pour récompenser les maîtres compagnons. Les prix étaient décernés chaque année. En 1889 le ministre du commerce et de l'industrie adressa à la Société centrale des architectes une médaille commémorative de la fondation Salleron « comme témoignage de son estime pour l'initiative qu'elle a prise en donnant des récompenses au personnel ouvrier et contremaître du bâtiment ${ }^{29} »$.

\section{La vie privée}

Devenu sous-inspecteur du Service d'architecture de la Ville de Paris, exerçant une profession honorable et des revenus assurés, Léon Salleron se marie à La Madeleine le 11 février 1852. Il épouse Stéphanie, Joséphine Anna Pezé, fille d'un ancien notaire à Troyes, décédé, et de Joséphine Argentin. Il a alors trente-et-un ans, la jeune fille vingt-quatre. Même s'il travaille depuis neuf ans, son apport personnel est modeste. Son contrat de mariage, signé chez $\mathrm{M}^{\mathrm{e}}$ Meunier le 28 janvier précédent, le crédite de deux mille francs en «habits, linges, hardes, bijoux et effets à son usage personnel, instruments de mathématiques et bibliothèque ». Ce sont ses oncles François et Louis Marcellot qui le dotent d'une somme de 25000 francs. Anna apporte, elle aussi, deux mille francs en « habits, linges, hardes et menus bijoux à son usage ». Sa mère la dote de 43000 francs en avancement d'hoirie et provenant de la succession de son père ${ }^{30}$. Deux filles vont naître au foyer, Augustine, Charlotte, Thérèse, le 21 octobre 1852, puis Charlotte, Hélène trois ans après, le 2 octobre 1855. Le ménage est sans histoire. Mais, huit ans plus tard, en décembre 1863, meurt de tuberculose à trente-sept ans, le dernier frère de Léon, Paul Salleron, qui laisse une jeune veuve de vingt-six ans et quatre petits enfants de quatre ans et demi, 3 ans, 2 ans et quatre mois. Léon est nommé subrogé tuteur de ses neveux. Il veillera avec soin sur leur éducation et aura pour élève René, le seul fils survivant de son 
frère. Il interviendra en sa faveur auprès de Bouvard, son ancien collègue devenu directeur du Service d'architecture, pour le faire entrer au Service d'architecture de la Ville de Paris. «L'oncle Léon » était une figure tutélaire de la famille de son neveu René et son souvenir évoqué avec dévotion. Son portrait par Le Chevallier Chevignard figurait en bonne place dans le cabinet de travail de René Salleron. C'est à René que Léon, sans descendance mâle, transmis les archives familiales de la famille Salleron.

Thérèse, la fille aînée de Léon, épousa à 20 ans Eugène Théodore Homberg le 17 octobre 1873 à Saint-Augustin. Le jeune marié, âgé de 27 ans, était un architecte, élève de Vaudremer, et le petit-fils d'un ingénieur des ponts-et-chaussées, autrefois auteur d'un rapport sur l'aménagement de la Bièvre, rivière sur les bords de laquelle était né son beau-père. Il devint l'associé de ce dernier et on leur doit de nombreuses réalisations en commun. Il y eut plusieurs architectes parmi leurs descendants.

Hélène, la plus jeune, épousa Pierre Bonnassieux le 18 septembre 1877 à Saint-Augustin. Elle avait 21 ans et lui 27. Pierre était le fils d'un sculpteur connu, grand-prix de Rome, auteur, en particulier, de la statue de la Vierge du Puy-en-Velay, fondue d'après son œuvre à partir de 213 canons de la bataille de Sébastopol. Pierre était archiviste paléographe, archiviste aux Archives nationales. Il mourut prématurément le 3 mai 1895, à 45 ans, laissant trois jeunes enfants.

Le 31 décembre 1884 Léon Salleron fut décoré de la Légion d'honneur.

Son activité d'architecte lui avait permis de se constituer une certaine fortune. Dès 1857 il achète une maison à Versailles, qu'il revendra en 1866 lorsqu'il achète des terrains pour faire construire. Comme ancien architecte de la Ville de Paris, il bénéficiait d'une rente annuelle de 4 067,72 francs Dans la maison de Versailles, au 6 avenue de Villeneuvel'Étang, qu'il avait fait construire, il vivait retiré, servi par une femme de chambre, aidée elle-même par une "ménagère ", une cuisinière et un jardinier, domesticité qu'il n'oublie pas dans son testament. Sa succession fait apparaître un actif de 629856 francs, qu'il laisse pour moitié à chacune de ses filles. Actif composé de valeurs mobilières, et d'immeubles, 15 et 17 rue Portalis et 130 boulevard Haussmann à Paris, qui étaient loués par appartements, et de sa maison avenue de Villeneuve-l'Étang à Versailles ${ }^{31}$. Il faut noter que cette fortune ne provenait pas exclusivement de son activité d'architecte car, comme ses frères et sœurs, Léon Salleron avait hérité de ses oncles Marcellot d'une somme de 270980 francs alors que ses parents n'avaient au contraire laissé aucune fortune à leur décès. 


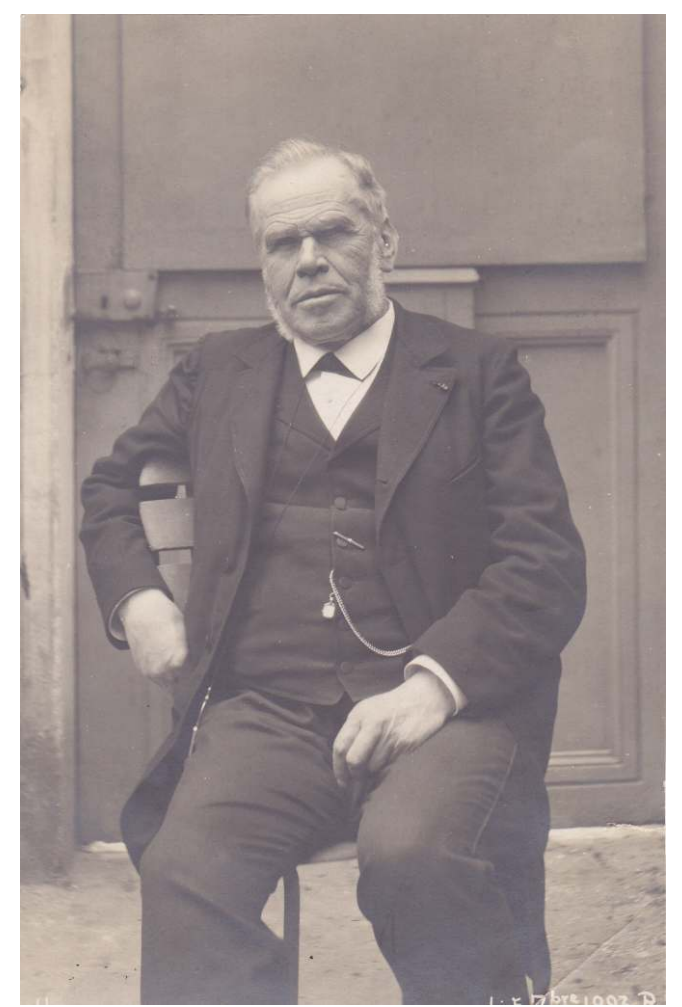

5-9-1893

Cl. de René Salleron

51 Il mourut le 19 novembre 1904 dans sa maison de Versailles, où il demeurait non loin de sa fille Hélène, veuve elle aussi. Il avait survécu 33 ans à sa femme, morte en 1871 à 44 ans.

Dans le discours prononcé sur sa tombe, Bartaumieux, vice-président de la Société centrale des architectes français, fait son éloge en soulignant : « Chez Léon Salleron, les qualités de l'homme n'étaient pas inférieures au mérite de l'artiste; il était d'un commerce absolument sûr, serviable pour tous ceux qui avaient affaire à lui, bienveillant et obligeant pour les jeunes, d'excellent conseil en toutes circonstances ${ }^{32}$. »

De son côté, dans sa notice nécrologique, Charles Lucas, après avoir rappelé ses fonctions et travaux, rapporte que, lors de son inhumation au Père-Lachaise le 23 novembre, le prêtre qui officiait a souligné que : «à l'exemple du Divin maître, M. Salleron avait traversé la vie en faisant le bien ${ }^{33}$.". Même en faisant la part du style hagiographique propre aux oraisons funèbres, il est possible de souscrire à ces jugements.

\section{ANNEXES}




\section{Chronologie}

29 décembre, naissance à Paris

1833 Manuel des salles d'asile.- J.D.M. Cochin

1836 Faillite de son père Augustin

1842 entre en $2^{\mathrm{e}}$ classe à l'École royale des beaux-arts

1843 entre dans le Service d'architecture de la Ville de Paris comme conducteur de travaux

1844 Diplôme d'ingénieur de l'École centrale des arts et manufactures

1845-1850 Sous-inspecteur

1852 Mariage avec Anna Pezé.

1853 Inspecteur du service ordinaire

1860 Annexion des communes autour de Paris. Haussmann réforme le Service de la Ville, création d'un corps d'architectes municipaux

1861 Projet pour l'Opéra de Paris, (Revue générale d'architecture et des travaux publics*1861)

Immeuble $61 \mathrm{Bd}$ Malesherbes, VIII (Daly*, archives CVP*, Goy-Truffaut)

1865 Chapelle de Clagny

La Mairie de Paris impose des prescriptions pour les maisons d'école

1867 Octave Gréard impose le même programme à toutes les écoles primaires

1867-1877 Mairie du XX', pl. Gambetta ( Narjoux*, Moniteur des architectes* 1878-79,

Hautecoeur t. VII, archives CVP*, expo « de Belleville à Charonne », dictionnaire monuments de Paris*)

1870 0ctave Gréard devient directeur de l'enseignement primaire de la Seine

1870 ? École élémentaire du 17 rue Boileau, XVI

Château de Blanchelande, Orne, pour Eugène Poriquet

1872 Appropriation à usage scolaire du 354 rue des Pyrénées, XXe ,id. 104 bd de Belleville ( DCM)

1873-1875 École élémentaire 14-16 rue Riblette, XXe (Narjoux*, expo «l'école primaire», Dictionnaire des monuments de Paris)

1873 Médaille à l'Exposition universelle de Vienne

1874 Hôtel Normant, Romorantin (Bulletin trimestriel Société d'art, d'histoire et d'archéologie de la Sologne, $\mathrm{n}^{\circ} 181,4^{\mathrm{e}}$ trimestre 2010, p. 77)

1875 Médaille à l'Exposition universelle de Londres

Appropriation à usage scolaire, école élémentaire 293 rue des Pyrénées XXe (DCM)

1876 Duval réorganise de Service d'architecture par type de bâtiments

Architecte en chef des bâtiments scolaires 
Agrandissement de l'école élémentaire du 62 rue Lepic, XVIII (expo «l'école primaire »)

École élémentaire du $75 \mathrm{Bd}$ de Belleville, $\mathrm{XI}^{\mathrm{e}}$ (Semaine des constructeurs Moniteur des

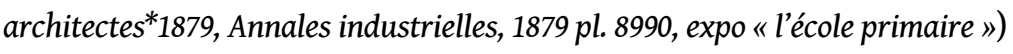

École élémentaire du 77 Bd de Belleville, $\mathrm{XI}^{\mathrm{e}}$

École élémentaire, 4 Place du commerce, $\mathrm{XV}^{\mathrm{e}} \underline{\text { à supprimer }}$

1876-1878 Asile Ville de Paris, 4 rue du Jourdain, $\mathrm{XX}^{\mathrm{e}}$ (expo «l'école primaire », Planat*, Moniteur des architectes* 1883)

1876-1879 École, 4 place du Commerce, $\mathrm{XV}^{\mathrm{e}}$ (expo «l'école primaire »)

1876-1882 École normale primaire d'instituteurs pour le dépt de la Seine, 10 rue Molitor, $\mathrm{XVI}^{\mathrm{e}}$ (Semaine des constructeurs, Narjoux*, Siguret, Hautecoeur t. VII)

1877 École élémentaire du 9 rue Blanche, IX ${ }^{\mathrm{e}}$ (Narjoux)

École élémentaire du 16 rue Julien Lacroix, $\mathrm{XX}^{\mathrm{e}}$

Annexe du théâtre de Biré, 101 rue de l'Université, $7^{\mathrm{e}}$ (Semaine des constructeurs)

Construction du passage Sainte-Marie, rue Paul-Louis Courrier ou rue de Saint-Simon, bd Saint-Germain, VII ${ }^{\mathrm{e}}$ (Semaine des constructeurs)

Asile, rue de la Municipalité, rue Chardon-Lagache, $\mathrm{XVI}^{\mathrm{e}}$ (Semaine des constructeurs)

1877-1878 École maternelle, 20 rue de Musset, $\mathrm{XVI}^{\mathrm{e}}$ (expo «l'école primaire »)

1878 Nouvelle organisation du Service d'architecture. On revient à l'arrondissement

La Loi Guizot ouvre des crédits affectés à la construction d'écoles

Agrandissement de l'école élémentaire du 21 rue Milton IX

École, rue Chardon-Lagache, $\mathrm{XVI}^{\mathrm{e}}$ (expo «l'école primaire »)

Annexes écoles d'apprentis de la Ville de Paris école Diderot, 62 Bd de la Villette XIX ${ }^{\mathrm{e}}$ ( Semaine des constructeurs)

École élémentaire du 2 rue Fessart, XIX (expo « l'école primaire »)

Asile pl. Saint-Pierre, rue Berthe, rue Tardieu, XVIII ${ }^{\mathrm{e}}$ (Semaine des constructeurs)

École élémentaire du 1 rue Foyatier, $18^{\mathrm{e}}$ (expo « l'école primaire »)

École élémentaire du 9 rue d'Argenteuil, $\mathrm{I}^{\mathrm{er}}$ (Semaine des constructeurs 1878)

Immeuble de rapport Lefebvre de la Vieuville, rue de Rivoli, rue Rouget de l'Isle, I $^{\text {er }}$ (S emaine des constructeurs 1878, 1879*)

Hôtels particuliers Villebesseyx, 47-55 rue Saint-Didier, $\mathrm{XVI}^{\mathrm{e}}$ (Semaine des constructeurs)

Construction Étienne de Malaincourt, 57 rue Saint-Didier, $\mathrm{XVI}^{\mathrm{e}}$ (Semaine des constructeurs)

Médaille à l'Exposition universelle de Paris pour la mairie du $\mathrm{XX}^{\mathrm{e}}$, les écoles du bd de Belleville, rue Riblette et l'asile rue du Jourdain

École, 9 rue Blanche, rue de la Trinité, IX (Semaine des constructeurs, expo «l'école primaire ", Narjoux*)

École de garçons, 1 rue Foyatier, XVIII (expo «l'école primaire ») 
1878-1882 École normale, rue Molitor (Narjoux)

1878 il construit pour lui l'immeuble du 15 rue Portalis VIII', et un hôtel particulier au 17, (Semaine des constructeurs)

1879, 30 mars membre de la Commission des bâtiments scolaires nouvellement créée

Annexe école rue Milton, $9^{e}$ (Semaine des constructeurs)

Reconstruction école, 16 rue Julien Lacroix XXe (expo «l'école primaire»)

Immeuble Filon, 69 avenue de la Grande Armée, XVI (Semaine des constructeurs 1879)

Retraite comme architecte de 1ère classe

1880, 17 juin règlement pour la construction des bâtiments scolaires

24 septembre membre du Comité des bâtiments scolaires nouvellement créé

Entre à la Société centrale des architectes

Médaille de la Société centrale des architectes pour travaux d'architecture privée

Immeuble Pinta, 4 rue Linné, $5^{\mathrm{e}}$ (Semaine des constructeurs)

1881 Jardin de l'atelier photo Plantet, 113 rue ND des Champs, VI (Semaine des constructeurs)

Surélévation cour $1^{\text {er }}$ étage, 115 bd Saint-Martin, III ${ }^{e}$ (Semaine des constructeurs)

Hôtel particulier Duboys d'Angers, bd Suchet XVI ${ }^{e}$ (Semaine des constructeurs)

Bâtiments pour les Messageries nationales, 32 avenue Claude Vellefaux, rue de Sambreet-Meuse, $\mathrm{X}^{\mathrm{e}}$ (Semaine des constructeurs)

1882 annexe immeuble cour (avec Homberg), 11 rue de Courcelles VIII ${ }^{e}$ (Semaine des constructeurs)

Annexe immeuble Limona, 96 rue latérale, XVIII ${ }^{e}$ (Semaine des constructeurs)

1884 Hôtel particulier Romaguera, 55-61 av. des Champs-Élysées, rue Pierre-Charon, VIII ${ }^{e}$ (Semaine des constructeurs)

Pavement de la cour des sœurs de Saint-Vincent-de-Paul, 22 rue Vandrezanne, XIII ${ }^{e}$

1885 Raccordement d'un immeuble de rapport rue Pierre Charon, $3^{\mathrm{e}}$ (Semaine des constructeurs)

1885/1886 Immeuble en collaboration avec Homberg ( $A P^{*}$, Construction moderne 1885, $C M^{*}$ $\left.1887-88^{*}\right)$

1886 Immeuble Gentil, 18 avenue Hoche, VIII ${ }^{e}$ (Semaine des constructeurs)

Immeuble Coutelet, 88 rue de Monceau, VIII (Semaine des constructeurs, La Construction moderne 1888*)

Immeuble Pinet, $42-44$ rue de Paradis, $\mathrm{X}^{\mathrm{e}}$ (Semaine des constructeurs)

Galerie des magasins Latour, 63 rue de Montorgueil, II (Semaine des constructeurs 1886)

1887 Immeuble avenue des Champs-Elysées, VIII (La Construction moderne)

Annexe du Théatre de Biré, 101 rue de l'Université, VII (Semaine des constructeurs, AP*) 
1889 Hôtel particulier Vve Gentil, 177 rue du Fbg Saint-Honoré, VIII ${ }^{\mathrm{e}}$ (Semaine des constructeurs)

Président de la Commission chargée d'éviter les projets dispendieux pour les écoles 1890 Hôtel Dujardin et Cie supprimer

1892 Immeuble Pinta, 18 rue Cuvier, $\mathrm{V}^{\mathrm{e}}$ (BMO)

Surélévation aile cour pour Henry, 61 rue Claude Bernard, $V^{\mathrm{e}}$ (BMO)

Transformation boutique en appartement (Lorrain) 14 rue de Logelbach, XVII ${ }^{\mathrm{e}}$ (BMO)

1894 il est choisi par le préfet pour faire partie du jury de l'école suite au legs Ledru-Rollin 1895 Chapelle de l'École Gerson avec Homberg, (Dictionnaire des monuments de Paris, p. 326 ; $\mathrm{BMO})$

1897 immeuble Carraby, 121 avenue des Champs Elysées et 20 rue Vernet, VIII ${ }^{\mathrm{e}}\left(\mathrm{AP}^{*}\right)$

1898 Construction d'une cour pour la Société de l'Externat de Madrid, 14 rue de Madrid VIII ${ }^{\mathrm{e}}$ avec la collaboration de Homberg, (BMO)

Construction $3^{\mathrm{e}}$ étage pour Société des chemins de fer économiques $4 \mathrm{c}$. de Londres, $\mathrm{IX}^{\mathrm{e}}($ BMO)

Extension de la mairie du XVIII ${ }^{\mathrm{e}}, 1$ rue Jules Joffrin 18e travaux commencés par Varcollier, 1888, (Dictionnaire des monuments historiques)

190419 novembre, mort à Versailles

s.d Hôtel particulier avenue Raphaël, XVI ${ }^{\mathrm{e}}$ (Planat*)

s.d. Tombeaux des familles Néron et Boutet, Père-Lachaise, (Architecture funéraire contemporaine)

s.d. Château de Saint-Voir, Allier (David de Penanrun)

Les références aux ouvrages contenant des mentions des constructions et des illustrations ou plans, signalés par une astérisque, sont principalement tirées du Dictionnaire par noms d'architectes des constructions élevées à Paris aux XIX ${ }^{e}$ et XX $X^{e}$ siècles.

\section{Principales sources}

\section{Archives}

- Académie d'architecture. Catalogue des collections, vol.1, 1750-1900. ํㅜ 446-448, 488.

- Archives de Paris, séries Vm, plans et Vo, permis de construire

- Archives nationales, série $\mathrm{AJ}^{52}$, École nationale des beaux-arts

- Musée Carnavalet, cabinet des arts graphiques

\section{Ouvrages}

- L'Architecture. Journal hebdomadaire de la Société centrale des architectes français Paris : Librairie des imprimeries réunies, 1889-1939. 
- Anne-Marie Chatelet. La Naissance de l'architecture scolaire, les écoles élémentaires parisiennes de 1870 à 1914, Paris, Honoré Champion, 1999.

- Anne-Marie Chatelet. Paris à l'école, qui a eu cette idée folle. Ouvrage publié à l'occasion de l'exposition...,inaugurée le 28 janvier 1993 au Pavillon de l'Arsenal, Éditions du Pavillon de l'Arsenal, 1993.

- La Construction moderne. Journal hebdomadaire illustré, art, théorie appliquée, pratique, génie civil, industrie du bâtiment, Paris, Dujardin, 1885

- César Daly. Architecture funéraire contemporaine, Paris, Ducher, 1871.

- César Daly, L'Architecture privée au XXe siècle, $1^{\text {ère }}$ série, nouvelles maisons de Paris et des environs, Paris, Ducher, 1870.

- David de Penanrun, Roux et Delaire. Les Architectes élèves des Beaux-arts 1793-1907, Paris, 1907.

- Dictionnaire par noms d'architectes des constructions élevées à Paris au XIXeet XXe siècle, période 1876-1899, t. IV, Paris, Service des travaux historiques, 1966. Notice 4360.

- L'École primaire à Paris, 1870-1914 [expositions mairies du XV et du II ${ }^{\mathrm{e}}$ arrondissements, 1985], Paris, Délégation à l'action artistique de la ville de Paris, 1985.

- Félix Narjoux. Paris, monuments élevés par la ville, 1850-1880. 1, Édifices administratifs, 2, Édifices consacrés à l'instruction publique, Paris, Vve A. Morel, 1883.

- Isabelle Parizet, Inventaire des immeubles parisiens datés et signés antérieurs à 1876, Paris, Rotonde de la Villette, 2001.

(Ville de Paris. Commission du vieux Paris. Cahiers de la Rotonde, 24)

- Revue générale de l'architecture et des travaux publics. Journal des architectes, des ingénieurs, des archéologues, des industriels et des propriétaires sous la direction de César Daly, Paris, Paulin et Hetzel, 1840-1888.

- La Semaine des constructeurs. Journal hebdomadaire illustré des travaux publics et privés, publié par M. César Daly,... M. Paul Planat, Paris, Librairie générale de l'architecture et des travaux publics, Ducher et Cie, 1876

\section{NOTES}

1. Léon est bien son prénom d'usage et celui dont il signe ses plans. Il apparaitt souvent sous le prénom de Claude dans les ouvrages et Mme Chatelet ne le cite jamais autrement. Il est parfois confondu avec son neveu René (1859-1942), également architecte de la Ville de Paris.

2. Écho de la littérature et des beaux-arts en France et à l'étranger, 1843, p. 95-96.

3. Académie d'architecture, Catalogue des collections, 1750-1900, vol. 1, n 446.

4. Revue générale de l'architecture et des travaux publics, 1884, $\mathrm{n}^{\circ} 8$, col. 381.

5. Alice Thomine, «L'œuvre des architectes municipaux », XXe arrondissement : la montagne à Paris. Exposition organisée par les Archives de Paris à la Mairie du XXe arrondissement, 22 décembre 1999-2 mars 2000, Paris, Action artistique de la Ville de Paris, 1999.

6. La Construction moderne, 1888, $\mathrm{n}^{\circ} 25,23$ juin, p. 434.

7. Arch. de Paris, V2 M1 1 et L'Architecture, 1889, n 32, p. 379. 
8. L'École primaire à Paris, Délégation à l'action artistique de la Ville de Paris, Paris, Imp. alençonnaise, 1985.

9. Académie d'architecture. Catalogue des collections 1750-1900, vol. 1 p. 242, cote 447.

10. Revue générale d'architecture, vol. XIX, 1861, pl. 35-36.

11. Arch. de Paris, V2 M1 1.

12. Félix Narjoux, Paris, monuments élevés par la Ville, 1850-1880, Paris, Vve A. Morel, 1883.

13. Thierry Halay, « La Mairie », Le XIX ${ }^{e}$ arrondissement : une cité nouvelle [exposition, mairie du XIX e arrondissement 1996], Paris, Délégation à l'action artistique de la Ville de Paris, 1996.

14. De Belleville à Charonne, promenade historique à travers le $X X^{e}$ arrondissement. Collections du Musée Carnavalet. Exposition organisée avec le concours de la Délégations artistique de la Ville de Paris... mars-mai 1979, Paris, 1979, p. 12.

15. Exposition universelle internationale de 1878 à Paris. Groupe I, classe 4. Rapport sur la section d'architecture par M. Vaudremer, Paris, Impr. nationale, 1880, p. 11.

16. César Daly, l'Architecture privée au XX $X^{e}$ siècle, $1^{\text {ère }}$ série, nouvelles maisons de Paris et des environs, 2 maisons à loyer, pl.2.

17. Isabelle Parizet, Immeubles datés et signés antérieurs à 1876. p. 91 et photographie $n^{\circ} 211$.

18. C'est par une erreur due à la Semaine des constructeurs, pas toujours précise, que le Dictionnaire par noms d'architectes, dans la notice 4360, donne le nom de propriétaire sous la forme Auboy d'Anger.

19. 19. La Construction moderne, $1887, \mathrm{n}^{\circ} 52-53,1888, \mathrm{n}^{\circ} 1$.

20. Op.cit., 1888, $\mathrm{n}^{\circ}$ 41,13 octobre, p. 7, 17-18, pl 1-2.

21. Paul Planat, Habitations particulières. $1^{\text {ère }}$ série : hôtels privés, Paris, Dujardin et $\mathrm{C}^{\mathrm{ie}}, \mathrm{p} .56-59, \mathrm{pl}$. 48-49.

22. David de Penanrun, Roux et Delaire, Les architectes élèves des Beaux-arts, 1793-1907, Paris, Librairie de « La Construction moderne », 1907.

23. Société d'art, d'histoire et d'archéologie de la Sologne. Bulletin trimestriel. Numéro spécial: usine et famille Normant, $4^{\mathrm{e}}$ trimestre 2010.

24. La Construction moderne, $1885, \mathrm{n}^{\circ} 13$, col. 290 et $\mathrm{n}^{\circ} 16$, col. 324.

25. Op.cit.,1897, n 13, 16 mai, p. 312.

26. Arch. nat., $\mathrm{F}^{19} 10686$ et Arch. de Paris V7 M 331.

27. BnF, $\left[4^{\circ} \mathrm{Lk}^{7} 12614\right.$.

28. La Construction moderne, $1890, n^{\circ} 45,8$ novembre, p. $49-50$ et $n^{\circ} 48,29$ novembre p. 92.

29. L'Architecture, $1889, \mathrm{n}^{\circ} 5$.

30. Arch. nat., ET/XCVI/786.

31. Arch. dép. des Yvelines, $9 \mathrm{Q} 6208$ Déclaration de succession de Claude-Augustin-Léon Salleron, 19 mai 1905.

32. L'Architecture, $\mathrm{n}^{\circ}$ 50, 10 décembre 1904, p. 453-454.

33. La Construction moderne, $1904, \mathrm{n}^{\circ} 50,10$ décembre, p. 131-132.

\section{RÉSUMÉS}

Claude Augustin Léon Salleron naquit le 29 décembre1820 à Paris, dans une famille de tanneurs. Il entre à l'École des beaux-arts en 1842 après avoir travaillé dans l'atelier de Duban. Parallèlement il suit les cours de l'École centrale des arts et manufactures et entre comme 
conducteur de travaux à la Ville de Paris où il fera toute sa carrière, prenant sa retraite en 1879 comme architecte de $1^{\text {ère }}$ classe. Considéré comme éclectique par Hautecoeur, il se rapproche cependant de l'école rationaliste dans ses constructions scolaires simples et fonctionnelles.

D'abord chargé du $20^{\mathrm{e}}$ arrondissement pour lequel il construit les nouvelles casernes d'octroi, quelques écoles et la nouvelle mairie, il devient responsable des bâtiments scolaires en 1877. À ce titre il est désormais considéré comme un véritable spécialiste de la question et participe à de nombreuses commissions et jurys. Outre son activité publique il réalise de nombreuses constructions pour une clientèle privée, hôtels particuliers et immeubles à loyers à Paris, châteaux et villas à Versailles et en province.

Il est récompensé de prix aux expositions universelles de 1873, 1875 et 1878 et par une médaille de la Société des architectes en 1880 pour ses travaux d'architecture privée, décoré de la Légion d'honneur en 1884.

Il mourut le 19 novembre 1904 à Versailles.

Claude Augustin Léon Salleron was born on the $29^{\text {th }}$ of December 1820 in Paris, in a tanner's family. He was admitted at the École des beaux-arts in 1842 after working in Duban's studio. At the same time, he took courses at the École centrale des arts et manufactures and was employed as a site manager by the city of Paris. He spent his entire career as an architect and retired in 1879. Hautecoeur regarded him as eclectic, but his academic constructions, simple and functional, are closer to the rationalist school.

His first commissions were for the $20^{\text {th }}$ arrondissement, where he built the new toll pavilions, schools and the city hall. He became the head of academic buildings in 1877 . He was often seen as a specialist and participated in committees and juries. Aside from his public activities, he built mansions and housing blocks in Paris, and castles and villas in Versailles and the provinces, for a private clientele.

He was awarded a medal at the Paris World's Fair in 1879, 1875 and 1878, and also received the Architects Society medals in 1880 for his private buildings. The French government awarded him the Legion of Honour in 1884.

He died in Versailles on the $19^{\text {th }}$ of November 1904.

Claude Augustin Léon Salleron war am 29. Dezember 1820 in Paris, als Sohn einer Gerberfamilie geboren.

Im 1842, studierte er in der französichen Kunsthochschule nach eine kurze Zeitarbeit in dem Dubanswerkstattkreis. Im gleichen Zeit, besuchte er die École centrale des arts et manufactures (die zentrale Kunst und Manufakturhochschule) und war für die Ausführung der Bauarbeiten der Stadt Paris verantwortlich. Dort, machte er seine ganze Karriere, bis zum 1879 und galt als einen bestimmten Meister der Architektur und bekam den höchsten Architekturorden. Hautecœur mochte seinen gemischten Stil hinweisen. Aber, die unkomplizierten und funktionellen Schulgebäude folgten einem rationalisten Trend. Zuerst unterstützte er den 20. Gemeindebezirk und baute die neuen Kasernen, einige Schulen und die neue Bürgermeisterei. Im Jahre 1877, war er von den Schulgebäuden verantwortlich geworden. Deshalb war er als einen guten Kenner der Architektur betrachtet. Er nahm verschiedenen Ausschussen teil. Darüber hinzufügt, eine reiche Produktion für Privatkunden : herrschaftlichen Stadthäuser und Miethäuser in Paris, Schlöße und Villa in Versailles und in dem ganzen Land. Er bekam mehreren Auszeichnungen während der Weltaustellungen des Jahres 1873, 1875 und 1878. Für dieses Werk, die Architekten Gesellschaf zeichnete inh mit einer Medaille,im Jahre 1880 aus. Er war Ritter der Ehrenlegion im Jahre 1884 geworden.

Er starb am 19. November 1904 in Versailles. 


\section{AUTEUR}

\section{GENEVIÈVE BOISARD}

Geneviève Boisard est née en 1931. Elle est archiviste-paléographe et conservateur général chargée d'inspection des bibliothèques honoraire. Après avoir travaillé à la bibliothèque centrale de prêt de la Seine-maritime, puis à l'Institut de recherche et d'histoire des textes (CNRS), elle est entrée à la Bibliothèque nationale où elle a exercé diverses responsabilités, chef de service des publications officielles, directeur du département des entrées françaises. Elle a quitté la Bibliothèque nationale pour la Bibliothèque Sainte-Geneviève, qu'elle a dirigée de 1987 à 1995. Elle a été nommée ensuite à l'inspection générale des bibliothèques. Au cours de sa carrière, elle a eu l'occasion de participer à de nombreuses réunions internationales en matière de normalisation de l'information. Geneviève Boisard, née Salleron, est l'arrière-petite-nièce de l'architecte Léon Salleron. 